\title{
COHOMOLOGICAL STRUCTURE OF THE MAPPING CLASS GROUP AND BEYOND
}

\author{
SHIGEYUKI MORITA
}

\begin{abstract}
In this paper, we briefly review some of the known results concerning the cohomological structures of the mapping class group of surfaces, the outer automorphism group of free groups, the diffeomorphism group of surfaces as well as various subgroups of them such as the Torelli group, the IA outer automorphism group of free groups, the symplectomorphism group of surfaces.

Based on these, we present several conjectures and problems concerning the cohomology of these groups. We are particularly interested in the possible interplays between these cohomology groups rather than merely the structures of individual groups. It turns out that, we have to include, in our considerations, two other groups which contain the mapping class group as their core subgroups and whose structures seem to be deeply related to that of the mapping class group. They are the arithmetic mapping class group and the group of homology cobordism classes of homology cylinders.
\end{abstract}

\section{INTRODUCTION}

We begin by fixing our notations for various groups appearing in this paper. Let $\Sigma_{g}$ denote a closed oriented surface of genus $g$ which will be assumed to be greater than or equal to 2 unless otherwise specified. We denote by Diff $+\Sigma_{g}$ the group of orientation preserving diffeomorphisms of $\Sigma_{g}$ equipped with the $C^{\infty}$ topology. The same group equipped with the discrete topology is denoted by $\operatorname{Diff}_{+}^{\delta} \Sigma_{g}$. The mapping class group $\mathcal{M}_{g}$ is the group of path components of Diff $+\Sigma_{g}$. The Torelli group, denoted by $\mathcal{I}_{g}$, is the subgroup of $\mathcal{M}_{g}$ consisting of mapping classes which act on the homology group $H_{1}\left(\Sigma_{g} ; \mathbb{Z}\right)$ trivially. Thus we have an

1991 Mathematics Subject Classification. Primary 57R20, 55R40, 32G15, 57N05, 57M99, 20J06; Secondary 57N10, 20F28, 17B40, 17B56, 57R32.

Key words and phrases. mapping class group, automorphism group of free group, moduli space of curves, outer space, tautological algebra, free Lie algebra, derivation algebra, symplectic group, homology cylinder, homology sphere, symplectomorphism group.

The author is partially supported by JSPS Grant 16204005 and 16654011 . 
extension

$$
1 \longrightarrow \mathcal{I}_{g} \longrightarrow \mathcal{M}_{g} \longrightarrow \operatorname{Sp}(2 g, \mathbb{Z}) \longrightarrow 1
$$

where $\operatorname{Sp}(2 g, \mathbb{Z})$ denotes the Siegel modular group. Choose an embedded disk $D \subset \Sigma_{g}$ and a base point $* \in D \subset \Sigma_{g}$. We denote by $\mathcal{M}_{g, 1}$ and $\mathcal{I}_{g, 1}$ (resp. $\mathcal{M}_{g, *}$ and $\mathcal{I}_{g, *}$ ) the mapping class group and the Torelli group relative to $D$ (resp. the base point $*$ ).

Next let $F_{n}$ denote a free group of rank $n \geq 2$. Let Aut $F_{n}$ (resp. Out $F_{n}$ ) denote the automorphism group (resp. outer automorphism group) of $F_{n}$. Let IAut (resp. IOut $_{n}$ ) denote the subgroup of Aut $F_{n}$ (resp. Out $F_{n}$ ) consisting of those elements which act on the abelianization $H_{1}\left(F_{n} ; \mathbb{Z}\right)$ of $F_{n}$ trivially. Thus we have an extension

$$
1 \longrightarrow \text { IOut }_{n} \longrightarrow \text { Out } F_{n} \longrightarrow \mathrm{GL}(n, \mathbb{Z}) \longrightarrow 1 \text {. }
$$

The fundamental group $\pi_{1}\left(\Sigma_{g} \backslash\right.$ Int $\left.D\right)$ is a free group of rank $2 g$. Fix an isomorphism $\pi_{1}\left(\Sigma_{g} \backslash \operatorname{Int} D\right) \cong F_{2 g}$. By a classical result of Dehn and Nielsen, we can write

$$
\mathcal{M}_{g, 1}=\left\{\varphi \in \operatorname{Aut} F_{2 g}, \varphi(\gamma)=\gamma\right\}
$$

where the element $\gamma$ is defined by

$$
\gamma=\left[\alpha_{1}, \beta_{1}\right] \cdots\left[\alpha_{g}, \beta_{g}\right]
$$

in terms of appropriate free generators $\alpha_{1}, \beta_{1}, \cdots, \alpha_{g}, \beta_{g}$ of $F_{2 g}$. Then we have the following commutative diagram

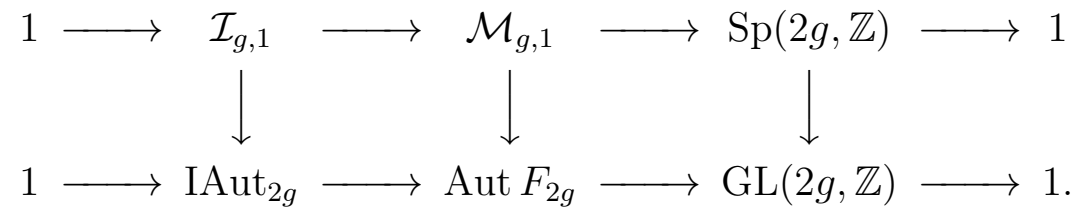

Similarly, for the case of the mapping class group with respect to a base point, we have

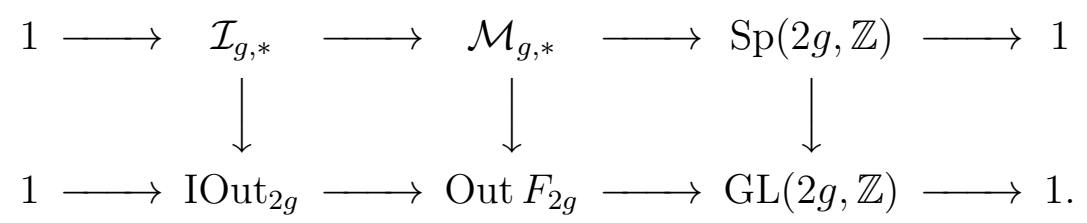

Next we fix an area form (or equivalently a symplectic form) $\omega$ on $\Sigma_{g}$ and we denote by Symp $\Sigma_{g}$ the subgroup of Diff $+\Sigma_{g}$ consisting of those elements which preserve the form $\omega$. Also let $\operatorname{Symp}_{0} \Sigma_{g}$ be the identity component of Symp $\Sigma_{g}$. Moser's theorem 84] implies that the quotient 
group Symp $\Sigma_{g} / \operatorname{Symp}_{0} \Sigma_{g}$ can be naturally identified with the mapping class group $\mathcal{M}_{g}$ and we have the following commutatvie diagram

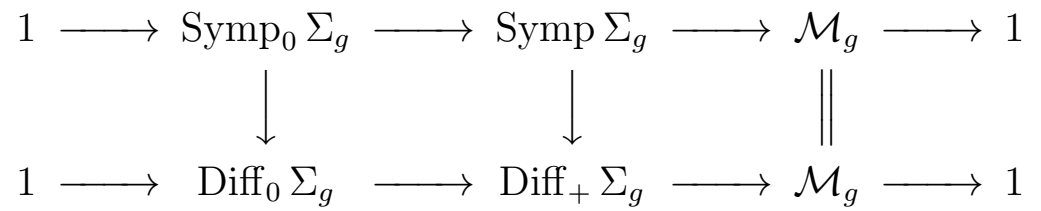

where $\operatorname{Diff}_{0} \Sigma_{g}$ is the identity component of Diff $+\Sigma_{g}$.

In this paper, we also consider two other groups. Namely the arithmetic mapping class group and the group of homology cobordism classes of homology cylinders. They will be mentioned in $\S 8$ and $\S 11$ respectively.

\section{TAUTOLOGiCAL ALGEBRA OF THE MAPPING CLASS GROUP}

Let $\mathbf{M}_{g}$ be the moduli space of smooth projective curves of genus $g$ and let $\mathcal{R}^{*}\left(\mathbf{M}_{g}\right)$ be its tautological algebra. Namely it is the subalgebra of the Chow algebra $\mathcal{A}^{*}\left(\mathbf{M}_{g}\right)$ generated by the tautological classes $\kappa_{i} \in$ $\mathcal{A}^{i}\left(\mathbf{M}_{g}\right)(i=1,2, \cdots)$ introduced by Mumford [85. Faber [15] made a beautiful conjecture about the structure of $\mathcal{R}^{*}\left(\mathbf{M}_{g}\right)$. There have been done many works related to and inspired by Faber's conjecture (we refer to survey papers 30 53 105. for some of the recent results including enhancements of Faber's original conjecture). However the most difficult part of Faber's conjecture, which claims that $\mathcal{R}^{*}\left(\mathbf{M}_{g}\right)$ should be a Poincaré duality algebra of dimension $2 g-4$, remains unsettled.

Here we would like to describe a topological approach to Faber's conjecture, in particular this most difficult part. For this, we denote by

$$
e_{i} \in H^{2 i}\left(\mathcal{M}_{g} ; \mathbb{Z}\right) \quad(i=1,2, \cdots)
$$

the $i$-th Mumford-Morita-Miller tautological class which was defined in 72 as follows. For any oriented $\Sigma_{g}$-bundle $\pi: E \rightarrow X$, the tangent bundle along the fiber of $\pi$, denoted by $\xi$, is an oriented plane bundle over the total space $E$. Hence we have its Euler class $e=\chi(\xi) \in$ $H^{2}(E ; \mathbb{Z})$. If we apply the Gysin homomorphism (or the integration along the fibers) $\pi_{*}: H^{*}(E ; \mathbb{Z}) \rightarrow H^{*-2}(X ; \mathbb{Z})$ to the power $e^{i+1}$, we obtain a cohomology class

$$
e_{i}(\pi)=\pi_{*}\left(e^{i+1}\right) \in H^{2 i}(X ; \mathbb{Z})
$$


of the base space $X$. By the obvious naturality of this construction, we obtain certain cohomology classes

$$
e \in H^{2}\left(\operatorname{EDiff}_{+} \Sigma_{g} ; \mathbb{Z}\right), \quad e_{i} \in H^{2 i}\left(\mathrm{BDiff}_{+} \Sigma_{g} ; \mathbb{Z}\right)
$$

where EDiff $+\Sigma_{g} \rightarrow$ BDiff $_{+} \Sigma_{g}$ denotes the universal oriented $\Sigma_{g}$-bundle. In the cases where $g \geq 2$, a theorem of Earle and Eells [12 implies that the two spaces EDiff $\Sigma_{+}$and BDiff $\Sigma_{+}$are Eilenberg-MacLane spaces $K\left(\mathcal{M}_{g, *}, 1\right)$ and $K\left(\mathcal{M}_{g}, 1\right)$ respectively. Hence we obtain the universal Euler class $e \in H^{2}\left(\mathcal{M}_{g, *} ; \mathbb{Z}\right)$ and the Mumford-Morita-Miller classes $e_{i} \in H^{2 i}\left(\mathcal{M}_{g} ; \mathbb{Z}\right)$ as group cohomology classes of the mapping class groups. It follows from the definition that, over the rationals, the class $e_{i}$ is the image of $(-1)^{i+1} \kappa_{i}$ under the natural projection $\mathcal{A}^{*}\left(\mathbf{M}_{g}\right) \rightarrow H^{*}\left(\mathcal{M}_{g} ; \mathbb{Q}\right)$.

Now we define $\mathcal{R}^{*}\left(\mathcal{M}_{g}\right)$ (resp. $\mathcal{R}^{*}\left(\mathcal{M}_{g, *}\right)$ ) to be the subalgebra of $H^{*}\left(\mathcal{M}_{g} ; \mathbb{Q}\right)\left(\right.$ resp. $\left.H^{*}\left(\mathcal{M}_{g, *} ; \mathbb{Q}\right)\right)$ generated by the classes $e_{1}, e_{2}, \cdots$ (resp. $\left.e, e_{1}, e_{2}, \cdots\right)$ and call them the tautological algebra of the mapping class group $\mathcal{M}_{g}$ (resp. $\mathcal{M}_{g, *}$ ). There is a canonical projection $\mathcal{R}^{*}\left(\mathbf{M}_{g}\right) \rightarrow \mathcal{R}^{*}\left(\mathcal{M}_{g}\right)$.

Let us denote simply by $H\left(\right.$ resp. $\left.H_{\mathbb{Q}}\right)$ the homology group $H_{1}\left(\Sigma_{g} ; \mathbb{Z}\right)$ $\left(\right.$ resp. $\left.H_{1}\left(\Sigma_{g} ; \mathbb{Q}\right)\right)$. Also we set

$$
U=\Lambda^{3} H / \omega_{0} \wedge H, \quad U_{\mathbb{Q}}=U \otimes \mathbb{Q}
$$

where $\omega_{0} \in \Lambda^{2} H$ denotes the symplectic class. $U_{\mathbb{Q}}$ is an irreducible representation of the algebraic group $\operatorname{Sp}(2 g, \mathbb{Q})$ corresponding to the Young diagram $\left[1^{3}\right]$ consisting of 3 boxes in a single column. Recall here that, associated to any Young diagram whose number of rows is less than or equal to $g$, there corresponds an irreducible representation of $\operatorname{Sp}(2 g, \mathbb{Q})($ cf. [20]). In our papers [77] 81], we constructed a morphism

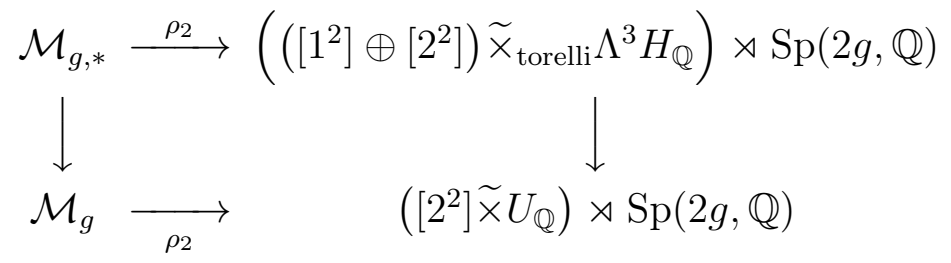

where $\left[2^{2}\right] \widetilde{x} U_{\mathbb{Q}}$ denotes a central extension of $U_{\mathbb{Q}}$ by $\left[2^{2}\right]$ corresponding to the unique copy $\left[2^{2}\right] \in H^{2}\left(U_{\mathbb{Q}}\right)$ and $\left(\left(\left[1^{2}\right] \oplus\left[2^{2}\right]\right) \widetilde{\times}_{\text {torelli }} \Lambda^{3} H_{\mathbb{Q}}\right.$ is defined similarly (see [1] for details). The diagram (6) induces the 
following commutative diagram.

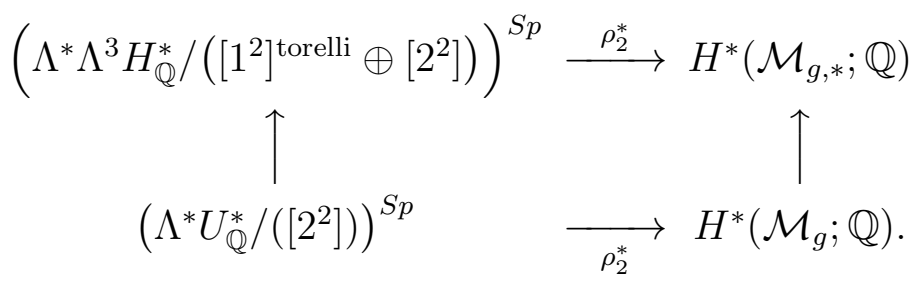

On the other hand, we proved in 51] 52] that the images of the above homomorphisms $\rho_{2}^{*}$ are precisely the tautological algebras. Here the concept of the generalized Morita-Mumford classes defined by Kawazumi 48 played an important role. Then in 82 , the effect of unstable degenerations of $S p$-modules appearing in (17) was analized and in particular a part of Faber's conjecture claiming that $\mathcal{R}^{*}\left(\mathcal{M}_{g}\right)$ is already generated by the classes $e_{1}, e_{2}, \cdots, e_{[g / 3]}$ was proved (later Ionel 39] proved this fact at the level of $\left.\mathcal{R}^{*}\left(\mathbf{M}_{g}\right)\right)$. Although the way of degenerations of $S p$-modules is by no means easy to be studied, it seems natural to expect the following.

Conjecture 1. The natural homomorphisms

$$
\left(\Lambda^{*} \Lambda^{3} H_{\mathbb{Q}}\right)^{S p} \rightarrow H^{*}\left(\mathcal{M}_{g, *} ; \mathbb{Q}\right), \quad\left(\Lambda^{*} U_{\mathbb{Q}}\right)^{S p} \rightarrow H^{*}\left(\mathcal{M}_{g} ; \mathbb{Q}\right)
$$

induce isomorphisms

$$
\begin{aligned}
\left(\Lambda^{*} \Lambda^{3} H_{\mathbb{Q}}^{*} /\left(\left[1^{2}\right]^{\text {torelli }} \oplus\left[2^{2}\right]\right)\right)^{S p} & \cong \mathcal{R}^{*}\left(\mathcal{M}_{g, *}\right) \\
\left(\Lambda^{*} U_{\mathbb{Q}}^{*} /\left(\left[2^{2}\right]\right)\right)^{S p} & \cong \mathcal{R}^{*}\left(\mathcal{M}_{g}\right) .
\end{aligned}
$$

Furthermore, the algebras on the left hand sides are Poincaré duality algebras of dimensions $2 g-2$ and $2 g-4$ respectively.

Here we mention that for a single Riemann surface $X$, the cohomology $H^{*}(\operatorname{Jac}(X) ; \mathbb{Q})$ is a Poincaré duality algebra of dimension $2 g$ while it can be shown that there exists a canonical isomorphism

$$
H^{*}(\operatorname{Jac}(X) ; \mathbb{Q}) /\left(\left[1^{2}\right]\right) \cong H^{*}(X ; \mathbb{Q})
$$

which is a Poincaré duality algebra of dimension 2. Here

$$
\left[1^{2}\right] \subset H^{2}(\operatorname{Jac}(X) ; \mathbb{Q})
$$

denotes the kernel $\operatorname{Ker}\left(\Lambda^{2} H_{\mathbb{Q}}^{*} \rightarrow \mathbb{Q}\right)$ of the intersection pairing and $\left(\left[1^{2}\right]\right)$ denotes the ideal generated by it. Observe that we can write $\Lambda^{*} U_{\mathbb{Q}}^{*}=$ $H^{*}\left(P H^{3}(\mathrm{Jac})\right)$ which is a Poincaré duality algebra of dimension $\left(\begin{array}{c}2 g \\ 3\end{array}\right)-$ $2 g$, where $P H^{3}$ (Jac) denotes the primitive part of the third cohomology 
of the Jacobian variety. Hence the above conjecture can be rewritten as

$$
\left(H^{*}\left(P H^{3}(\mathrm{Jac})\right) /\left(\left[2^{2}\right]\right)\right)^{S p} \cong \mathcal{R}^{*}\left(\mathcal{M}_{g}\right)
$$

so that it could be phrased as the family version of the above simple fact for a single Riemann surface.

\section{Higher GeOMEtRY OF THE MAPPING ClASS GROUP}

Madsen and Weiss 64 recently proved a remarkable result about the homotopy type of the classifying space of the stable mapping class group. As a corollary, they showed that the stable rational cohomology of the mapping class group is isomorphic to the polynomial algebra generated by the Mumford-Morita-Miller classes

$$
\lim _{g \rightarrow \infty} H^{*}\left(\mathcal{M}_{g} ; \mathbb{Q}\right) \cong \mathbb{Q}\left[e_{1}, e_{2}, \cdots\right]
$$

We also would like to mention fundamental results of Tillmann 104 and Madsen and Tillmann [63].

As was explained in [80], the classes $e_{i}$ serve as the (orbifold) Chern classes of the tangent bundle of the moduli space $\mathbf{M}_{g}$ and it may appear that, stably and quantitatively, the moduli space $\mathbf{M}_{g}$ is similar to the classifying space of the unitary group, namely the complex Grassmannian. However, qualitatively the situation is completely different and the moduli space has much deeper structure than the Grassmannian. Here we would like to present a few problems concerning "higher geometry" of the mapping class group where we understand the MumfordMorita-Miller classes as the primary characteristic classes.

First we recall the following problem, because of its importance, which was already mentioned in [80] (Conjecture 3.4).

Problem 2. Prove (or disprove) that the even Mumford-Morita-Miller classes $e_{2 i} \in H^{4 i}\left(\mathcal{I}_{g} ; \mathbb{Q}\right)$ are non-trivial, in a suitable stable range, as cohomology classes of the Torelli group.

The difficulty of the above problem comes from the now classical fact, proved by Johnson [40, that the abelianization of the Torelli group is very big, namely $H_{1}\left(\mathcal{I}_{g} ; \mathbb{Q}\right) \cong U_{\mathbb{Q}}(g \geq 3)$. Observe that if $\mathcal{I}_{g}$ were perfect, then the above problem would have been easily solved by simply applying the Quillen plus construction to each group of the group extension (11) and then looking at the homotopy exact sequence of the resulting fibration. The work of Igusa [38] (in particular Corollary 
8.5.17) shows a close connection between the above problem with another very important problem (see Problem [1] in $\S 4$ ) of non-triviality of Igusa's higher Franz-Reidemeister torsion classes in $H^{4 i}\left(\mathrm{IOut}_{n} ; \mathbb{R}\right)$ (Igusa uses the notation $\mathrm{Out}^{h} F_{n}$ for the group $\mathrm{IOut}_{n}$ ). We also refer to a recent work of Sakasai 95. which is related to the above problem.

Next we recall the following two well-known problems about the structure of the Torelli group which are related to a foundational work of Hain [29].

Problem 3. Determine whether the Torelli group $\mathcal{I}_{g}(g \geq 3)$ is finitely presentable or not (note that $\mathcal{I}_{g}(g \geq 3)$ is known to be finitely generated by Johnson 42]).

Problem 4. Let $\mathfrak{u}_{g}$ denote the graded Lie algebra associated to the prounipotent radical of the relative Malcev completion of $\mathcal{I}_{g}$ defined by Hain [29] and let $\mathfrak{u}_{g} \rightarrow \mathfrak{h}_{g}^{\mathbb{Q}}$ be the natural homomorphism (here $\mathfrak{h}_{g}^{\mathbb{Q}}$ denotes the graded Lie algebra consisting of symplectic derivations, with positive degrees, of the Malcev Lie algebra of $\pi_{1} \Sigma_{g}$ ). Determine whether this homomorphism is injective or not.

In [80, we defined a series of secondary characteristic classes for the mapping class group. However there was ambiguity coming from possible odd dimensional stable cohomology classes of the mapping class group. Because of the result of Madsen-Weiss cited above, we can now eliminate the ambiguity and give a precise definition as follows. For each $i$, we constructed in [51]52 explicit group cocycles $z_{i} \in Z^{2 i}\left(\mathcal{M}_{g} ; \mathbb{Q}\right)$ which represent the $i$-th Mumford-Morita-Miller class $e_{i}$ by making use of the homomorphism $\mathcal{M}_{g} \rightarrow U \rtimes \operatorname{Sp}(2 g, \mathbb{Z})$ constructed in [77] which extends the (first) Johnson homomorphism $\mathcal{I}_{g} \rightarrow U$. These cocycles are $\mathcal{M}_{g}$-invariant by the definition. Furthermore we proved that such cocycles are unique up to coboundaries. On the other hand, as is well known, any odd class $e_{2 i-1}$ comes from the Siegel modular group $\operatorname{Sp}(2 g, \mathbb{Z})$ so that there is a cocycle $z_{2 i-1}^{\prime} \in Z^{4 i-2}\left(\mathcal{M}_{g} ; \mathbb{Q}\right)$ which comes from $\operatorname{Sp}(2 g, \mathbb{Z})$. This cocycle is uniquely defined up to coboundaries and $\mathcal{M}_{g}$-invariant. Now consider the difference $z_{2 i-1}-z_{2 i-1}^{\prime}$. It is a coboundary so that there exists a cochain $y_{i} \in C^{4 i-3}\left(\mathcal{M}_{g} ; \mathbb{Q}\right)$ such that $\delta y_{i}=z_{2 i-1}-z_{2 i-1}^{\prime}$. Since $H^{4 i-3}\left(\mathcal{M}_{g} ; \mathbb{Q}\right)=0$ by [64] (in a suitable stable range), the cochain $y_{i}$ is well-defined up to coboundaries.

Now let $\mathcal{K}_{g}$ be the kernel of the Johnson homomorphism so that we have an extension

$$
1 \longrightarrow \mathcal{K}_{g} \longrightarrow \mathcal{I}_{g} \longrightarrow U \longrightarrow 1 \text {. }
$$

Recall that Johnson [43] proved that $\mathcal{K}_{g}$ is the subgroup of $\mathcal{M}_{g}$ generated by Dehn twists along separating simple closed curves on $\Sigma_{g}$. The 
cocycle $z_{2 i-1}^{\prime}$ is trivial on the Torelli group $\mathcal{I}_{g}$ while the cocycle $z_{2 i-1}$ (in fact any $z_{i}$ ) vanishes on $\mathcal{K}_{g}$. It follows that the restriction of the cochain $y_{i}$ to $\mathcal{K}_{g}$ is a cocycle. Hence we obtain a cohomology class

$$
d_{i}=\left[\left.y_{i}\right|_{\mathcal{K}_{g}}\right] \in H^{4 i-3}\left(\mathcal{K}_{g} ; \mathbb{Q}\right) .
$$

This cohomology class is $\mathcal{M}_{g}$-invariant where $\mathcal{M}_{g}$ acts on $H^{*}\left(\mathcal{K}_{g} ; \mathbb{Q}\right)$ via outer conjugations. This can be shown as follows. For any element $\varphi \in \mathcal{M}_{g}$, let $\varphi_{*}\left(y_{i}\right)$ be the cochain obtained by applying the conjugation by $\varphi$ on $y_{i}$. Since both cocycles $z_{2 i-1}, z_{2 i-1}^{\prime}$ are $\mathcal{M}_{g}$-invariant, we have $\delta \varphi_{*}\left(y_{i}\right)=\delta y_{i}$. Hence $\varphi_{*}\left(y_{i}\right)-y_{i}$ is a cocycle of $\mathcal{M}_{g}$. By the result of 64] again, we see that $\varphi_{*}\left(y_{i}\right)-y_{i}$ is a coboundary. Hence the restrictions of $\varphi_{*}\left(y_{i}\right)$ and $y_{i}$ to $\mathcal{K}_{g}$ give the same cohomology class.

Definition 5. We call the cohomology classes $d_{i} \in H^{4 i-3}\left(\mathcal{K}_{g} ; \mathbb{Q}\right)^{\mathcal{M}_{g}}$ $(i=1,2 \cdots)$ obtained above the secondary characteristic classes of the mapping class group.

The secondary classes $d_{i}$ are stable in the following sense. Namely the pull back of them in $H^{4 i-3}\left(\mathcal{K}_{g, 1} ; \mathbb{Q}\right)$ are independent of $g$ under natural homomorphisms induced by the inclusions $\mathcal{K}_{g, 1} \rightarrow \mathcal{K}_{g+1,1}$ where $\mathcal{K}_{g, 1}$ denotes the subgroup of $\mathcal{M}_{g, 1}$ generated by Dehn twists along separating simple closed curves on $\Sigma_{g} \backslash D$. This is because the cocycles $z_{2 i-1}, z_{2 i-1}^{\prime}$ are stable with respect to $g$. It follows that the secondary classes $d_{i}$ are defined for all $g$ as elements of $H^{4 i-3}\left(\mathcal{K}_{g, 1} ; \mathbb{Q}\right)^{\mathcal{M}_{g, 1}}$ although we have used the result of 64 , which is valid only in a stable range. However as elements of $H^{4 i-3}\left(\mathcal{K}_{g} ; \mathbb{Q}\right)^{\mathcal{M}_{g}}$ the class $d_{i}$ is defined only for $g \geq 12 i-9$ at present, although it is highly likely that it is defined for all $g$. It was proved in 75 that $d_{1}$ is the generator of $H^{1}\left(\mathcal{K}_{g} ; \mathbb{Z}\right)^{\mathcal{M}_{g}} \cong \mathbb{Z}$ for all $g \geq 2$. See 79 for another approach to the secondary classes.

Problem 6. Prove that all the secondary classes $d_{2}, d_{3}, \cdots$ are nontrivial.

Here is a problem concerning the first class $d_{1}$. Let $C$ be a separating simple closed curve on $\Sigma_{g}$ which divides $\Sigma_{g}$ into two compact surfaces of genera $h$ and $g-h$ and let $\tau_{C} \in \mathcal{K}_{g}$ be the Dehn twist along $C$. Then we know that the value of $d_{1}$ on $\tau_{C} \in \mathcal{K}_{g}$ is $h(g-h)$ (up to a constant depending on $g$ ). This is a very simple formula. However at present there is no known algorithm to calculate the value $d_{1}(\varphi)$ for a given element $\varphi \in \mathcal{K}_{g}$, say by analyzing the action of $\varphi$ on $\pi_{1} \Sigma_{g}$.

Problem 7. Find explicit way of calculating $d_{1}(\varphi)$ for any given element $\varphi \in \mathcal{K}_{g}$. In particular, determine whether the Magnus representation $\mathcal{I}_{g, 1} \rightarrow \mathrm{GL}(2 g ; \mathbb{Z}[H])$ of the Torelli group detects $d_{1}$ or not. 
Suzuki [103] proved that the Magnus representation of the Torelli group mentioned above is not faithful so that it may happen that the intersection of the kernel of the Magnus representation with $\mathcal{K}_{g}$ is not contained in the kernel of $d_{1}$. We may also ask whether the representation of the hyperelliptic mapping class group given by Jones [45], restricted to the intersection of this group with $\mathcal{K}_{g}$, detects $d_{1}$ or not (cf. Kasahara [46] for a related work for the case $g=2$ ). There are also various interesting works related to the class $d_{1}$ such as Endo [14 and Morifuji [7] treating the hyperelliptic mapping class group, Kitano 54] as well as Hain and Reed [33.

Recently Biss and Farb [5] proved that the group $\mathcal{K}_{g}$ is not finitely generated for all $g \geq 3\left(\mathcal{K}_{2}\right.$ is known to be an infinitely generated free group by Mess [69). However it is still not yet known whether the abelianization $H_{1}\left(\mathcal{K}_{g}\right)$ is finitely generated or not (cf. Problem 2.2 of [80]).

Finally we would like to mention that Kawazumi [50] is developing a theory of harmonic Magnus expansions which gives in particular a system of differential forms representing the Mumford-Morita-Miller classes on the universal family of curves over the moduli space $\mathbf{M}_{g}$.

\section{Outer Automorphism Group of FreE Groups}

As already mentioned in $\S 1$, let $F_{n}$ denote a free group of rank $n \geq 2$ and let Out $F_{n}=$ Aut $F_{n} / \operatorname{Inn} F_{n}$ denote the outer automorphism group of $F_{n}$. In 1986, Culler and Vogtmann [11] defined a space $X_{n}$, called the Outer Space, which plays the role of the Teichmüller space where the mapping class group is replaced by Out $F_{n}$. In particular, they proved that $X_{n}$ is contractible and Out $F_{n}$ acts on it properly discontinuously. The quotient space

$$
\mathbf{G}_{n}=X_{n} / \text { Out } F_{n}
$$

is called the moduli space of graphs which is the space of all the isomorphism classes of metric graphs with fundamental group $F_{n}$. Recently many works have been done on the structure of Out $F_{n}$ as well as $\mathbf{G}_{n}$, notably by Vogtmann (see her survey paper [106]), Bestvina (see [2]) and many others.

It is an interesting and important problem to compare similarity as well as difference between the mapping class group and Out $F_{n}$ which will be discussed at several places in this book. Here we would like to concentrate on the cohomological side of this problem. 
Hatcher and Vogtmann [37] (see also Hatcher [35]) proved that the homology of Out $F_{n}$ stabilizes in a certain stable range. This is an analogue of Harer's stability theorem 34 for the mapping class group. More precisely, they proved that the natural homomorphisms

$$
\text { Aut } F_{n} \rightarrow \text { Aut } F_{n+1}, \quad \text { Aut } F_{n} \rightarrow \text { Out } F_{n}
$$

induce isomorphisms on the $i$-dimensional homology group for $n \geq$ $2 i+2$ and $n \geq 2 i+4$, respectively. Thus we can speak of the stable cohomology group

$$
\lim _{n \rightarrow \infty} \widetilde{H}^{*}\left(\operatorname{Out} F_{n}\right)
$$

of Out $F_{n}$.

In the case of the mapping class group, it was proved in [70] 73 that the natural homomorphism $\mathcal{M}_{g} \rightarrow \operatorname{Sp}(2 g, \mathbb{Z})$ induces an injection

$$
\lim _{g \rightarrow \infty} H^{*}(\operatorname{Sp}(2 g, \mathbb{Z}) ; \mathbb{Q}) \cong \mathbb{Q}\left[c_{1}, c_{3}, \cdots\right] \subset \lim _{g \rightarrow \infty} H^{*}\left(\mathcal{M}_{g} ; \mathbb{Q}\right)
$$

on the stable rational cohomology group where the stable rational cohomology of $\operatorname{Sp}(2 g, \mathbb{Z})$ was determined by Borel [6] [7. In the case of Out $F_{n}$, Igusa proved the following remarkable result which shows a sharp contrast with the case of the mapping class group (see Theorem 8.5.3 and Remark 8.5.4 of [38]).

Theorem 8 (Igusa[38]). The homomorphism

$$
\widetilde{H}^{k}(\mathrm{GL}(n, \mathbb{Z}) ; \mathbb{Q}) \longrightarrow \widetilde{H}^{k}\left(\text { Out } F_{n} ; \mathbb{Q}\right)
$$

induced by the natural homomorphism Out $F_{n} \rightarrow \mathrm{GL}(n, \mathbb{Z})$ is the 0-map in the stable range $n \geq 2 k+1$.

Recall here that the stable cohomology of $\operatorname{GL}(n, \mathbb{Z})$ is given by

$$
\lim _{n \rightarrow \infty} H^{*}(\mathrm{GL}(n, \mathbb{Z}) ; \mathbb{Q}) \cong \Lambda_{\mathbb{Q}}\left(\beta_{5}, \beta_{9}, \beta_{13}, \cdots\right)
$$

due to Borel in the above cited papers.

On the other hand, the first non-trivial rational cohomology of the group Aut $F_{n}$ was given by Hatcher and Vogtmann 36. They showed that, up to cohomology degree 6 , the only non-trivial rational cohomology is

$$
H^{4}\left(\text { Aut } F_{4} ; \mathbb{Q}\right) \cong \mathbb{Q} .
$$

Around the same time, by making use of a remarkable theorem of Kontsevich given in [55] [56], the author constructed many homology classes in $H_{*}\left(\right.$ Out $\left.F_{n} ; \mathbb{Q}\right)$ (see 80 and $\S 10$ below). The simplest one in this construction gave a series of elements

$$
\mu_{i} \in H_{4 i}\left(\text { Out } F_{2 i+2} ; \mathbb{Q}\right) \quad(i=1,2, \cdots)
$$


and the first one $\mu_{1}$ was shown to be non-trivial by a computer calculation. Responding to an inquiry of the author, Vogtmann communicated us that she modified the argument in 36] to obtain an isomorphism $H^{4}\left(\right.$ Out $\left.F_{4} ; \mathbb{Q}\right) \cong \mathbb{Q}$. Thus we could conclude that $\mu_{1}$ is the generator of this group (see [80]106]). Recently Conant and Vogtmann proved that the second class $\mu_{2} \in H_{8}\left(\right.$ Out $\left.F_{6} ; \mathbb{Q}\right)$ is also non-trivial in their paper [10] where they call $\mu_{i}$ the Morita classes. Furthermore they constructed many cycles of the moduli space $\mathbf{G}_{n}$ of graphs by explicit constructions in the Outer Space $X_{n}$.

More recently, Ohashi 91 determined the rational cohomology group of Out $F_{n}$ for all $n \leq 6$ and in particular he showed

$$
H_{8}\left(\text { Out } F_{6} ; \mathbb{Q}\right) \cong \mathbb{Q} \text {. }
$$

It follows that $\mu_{2}$ is the generator of this group. At present, the above two groups (and one more group, $H_{7}\left(\right.$ Aut $\left.F_{5} ; \mathbb{Q}\right) \cong \mathbb{Q}$ proved by Gerlits 25]) are the only known non-trivial rational homology groups of Out $F_{n}$ (and Aut $F_{n}$ ). Now we would like to present the following conjecture based on our expectation that the classes $\mu_{i}$ should concern not only the cohomology of Out $F_{n}$ but also the structure of the arithmetic mapping class group (see §8) as well as homology cobordism invariants of homology 3 -spheres as will be explained in $\S 11$ below and [83].

Conjecture 9. The classes $\mu_{i}$ are non-trivial for all $i=1,2, \cdots$.

More generally we have the following.

Problem 10. Produce non-trivial rational (co)homology classes of Out $F_{n}$.

Next we consider the group IOut $_{n}$. In 38] Igusa defined higher FranzReidemeister torsion classes

$$
\tau_{2 i} \in H^{4 i}\left(\text { IOut }_{n} ; \mathbb{R}\right)
$$

as a special case of his general theory. These classes reflect Igusa's result mentioned above (Theorem 8) that the pull back of the Borel classes $\beta_{4 i+1} \in H^{4 i+1}(\mathrm{GL}(n, \mathbb{Z}) ; \mathbb{R})$ in $H^{4 i+1}\left(\right.$ Out $\left.F_{n} ; \mathbb{R}\right)$ vanish. However it seems to be unknown whether his classes are non-trivial or not.

Problem 11 (Igusa). Prove that the higher Franz-Reidemeister torsion classes $\tau_{2 i} \in H^{4 i}\left(\right.$ IOut $\left._{n} ; \mathbb{R}\right)$ are non-trivial in a suitable stable range.

In the unstable range, where the Borel classes vanish in $H^{*}(\mathrm{GL}(n, \mathbb{Z}) ; \mathbb{R})$, there seem to be certain relations between the classes $\tau_{2 i}$, (dual of) $\mu_{i}$ and unstable cohomology classes in $H^{*}(\mathrm{GL}(n, \mathbb{Z}) ; \mathbb{Q})$. As the first such example, we would like to ask the following specific problem. 
Problem 12. Prove (or disprove) that the natural homomorphism

$$
H^{4}\left(\mathrm{Out} F_{4} ; \mathbb{Q}\right) \cong \mathbb{Q} \longrightarrow H^{4}\left(\mathrm{IOut}_{4} ; \mathbb{Q}\right)^{G L}
$$

is an isomorphism where the right hand side is generated by (certain non-zero multiple of) $\tau_{2}$.

Here is another very specific problem. We know the following groups explicitly by various authors:

$$
\begin{aligned}
& H^{8}\left(\mathcal{M}_{3, *} ; \mathbb{Q}\right)\left.\cong \mathbb{Q}^{2} \quad(\text { Looijenga } 61]\right) \\
& H^{8}(\mathrm{GL}(6, \mathbb{Z}) ; \mathbb{Q}) \cong \mathbb{Q} \quad(\text { Elbaz-Vincent, Gangl, Soulé [13] }) \\
& H^{8}\left(\text { Out } F_{6} ; \mathbb{Q}\right) \cong \mathbb{Q} \quad(\text { Ohashi }[91])
\end{aligned}
$$

On the other hand, we have the following natural injection $i$ as well as projection $p$

$$
\mathcal{M}_{3, *} \stackrel{i}{\longrightarrow} \text { Out } F_{6} \stackrel{p}{\longrightarrow} \mathrm{GL}(6, \mathbb{Z}) .
$$

Problem 13. Determine the homomorphisms

$$
H^{8}\left(\mathcal{M}_{3, *} ; \mathbb{Q}\right) \stackrel{i^{*}}{\longleftarrow} H^{8}\left(\operatorname{Out} F_{6} ; \mathbb{Q}\right) \stackrel{p^{*}}{\longleftarrow} H^{8}(\mathrm{GL}(6, \mathbb{Z}) ; \mathbb{Q})
$$

induced by the above homomorphisms in (9).

Remark 14. It seems to be natural to conjecture that the right map in (10) is an isomorphism while the left map is trivial. The former part is based on a consideration of possible geometric meaning of the classes $\mu_{i} \in H_{4 i}\left(\right.$ Out $\left.F_{2 i+2} ; \mathbb{Q}\right)$. For the particular case $i=2$ here, it was proved in 13 that $H^{9}(\mathrm{GL}(6, \mathbb{Z}) ; \mathbb{Q})=0$. It follows that the Borel class in $H^{9}(\mathrm{GL}(6, \mathbb{Z}) ; \mathbb{R})$ vanishes. Because of this, it is likely that the Igusa class $\tau_{4} \in H^{8}\left(\mathrm{IOut}_{6} ; \mathbb{R}\right)$ would vanish as well and the class $\mu_{2}$ would survive in $H_{8}(\mathrm{GL}(6, \mathbb{Z}) ; \mathbb{Q})$. For the latter part, see Remark 19 below.

Problem 15. Define unstable (co)homology classes of $\operatorname{GL}(n, \mathbb{Z})$. In particular, what can be said about the image of $\mu_{i} \in H_{4 i}\left(\right.$ Out $\left.F_{2 i+2} ; \mathbb{Q}\right)$ in $H_{4 i}(\mathrm{GL}(2 i+2, \mathbb{Z}) ; \mathbb{Q})$ under the projection Out $F_{2 k+2} \rightarrow \mathrm{GL}(2 k+2, \mathbb{Z})$ ?

The above known results as well as explicit computation made so far seem to support the following conjecture (which might be something like a folklore).

Conjecture 16. The stable rational cohomology of Out $F_{n}$ is trivial. Namely

$$
\lim _{n \rightarrow \infty} \widetilde{H}^{*}\left(\operatorname{Out} F_{n} ; \mathbb{Q}\right)=0 .
$$

We can aslo ask how the cohomology of Out $F_{n}$ with twisted coefficients look like. 
Problem 17. Compute the cohomology of Aut $F_{n}$ and Out $F_{n}$ with coefficients in various $\operatorname{GL}(n, \mathbb{Q})$-modules.

For example, we could ask how Looijenga's result 62 for the case of the mapping class group can be generalized in these contexts. We refer to the work of Kawazumi [49] and also Satoh [97] for recent results concerning the above problem.

Finally we recall the following well known problem.

Problem 18. Determine whether the natural homomorphisms

$$
\begin{aligned}
& \widetilde{H}^{*}\left(\text { Aut } F_{2 g} ; \mathbb{Q}\right) \longrightarrow \widetilde{H}^{*}\left(\mathcal{M}_{g, 1} ; \mathbb{Q}\right) \\
& \widetilde{H}^{*}\left(\operatorname{Out} F_{2 g} ; \mathbb{Q}\right) \longrightarrow \widetilde{H}^{*}\left(\mathcal{M}_{g, *} ; \mathbb{Q}\right)
\end{aligned}
$$

induced by the inclusions $\mathcal{M}_{g, 1} \rightarrow$ Aut $F_{2 g}, \mathcal{M}_{g, *} \rightarrow$ Out $F_{2 g}$ are trivial or not.

We refer to a result of Wahl [107] for a homotopy theoretical property of the homomorphism $\mathcal{M}_{g, 1} \rightarrow$ Aut $F_{2 g}$ where $g$ tends to $\infty$.

Remark 19. The known results as well as explicit computations made so far seem to suggest that the above maps are trivial. According to a theorem of Kontsevich [55] [56] (see $\S 9$ below), the triviality of the second map above is equivalent to the statement that the natural inclusion

$$
\mathfrak{l}_{\infty}^{+} \longrightarrow \mathfrak{a}_{\infty}^{+}
$$

between two infinite dimentional Lie algebras (see $\S 9$ for the definition) induces the trivial map

$$
H^{*}\left(\mathfrak{a}_{\infty}^{+}\right)^{S p} \longrightarrow H^{*}\left(\mathfrak{l}_{\infty}^{+}\right)^{S p}
$$

in the $S p$-invariant cohomology groups. Here the trivial map means that it is the 0-map except for the bigraded parts which correspond to the 0-dimensional homology groups of $\mathcal{M}_{g, *}$ and Out $F_{2 g}$.

Remark 20. In this paper, we are mainly concerned with the rational cohomology group of the mapping class group, Out $F_{n}$ and other groups. As for cohomology group with finite coefficients or torsion classes, here we only mention the work of Galatius 22 which determines the mod $p$ stable cohomology of the mapping class group and also Hatcher's result [35] that the stable homology of Out $F_{n}$ contains the homology of $\Omega^{\infty} S^{\infty}$ as a direct summand. 


\section{The Derivation Algebra of FReE Lie Algebras AND the}

TRACES

As in $\S 1$, let $F_{n}$ be a free group of rank $n \geq 2$ and let us denote the abelianization $H_{1}\left(F_{n}\right)$ of $F_{n}$ simply by $H_{n}$. Also let $H_{n}^{\mathbb{Q}}=H_{n} \otimes \mathbb{Q}$. Sometimes we omit $n$ and we simply write $H$ and $H_{\mathbb{Q}}$ instead of $H_{n}$ and $H_{n}^{\mathbb{Q}}$. Let

$$
\mathcal{L}_{n}=\oplus_{k=1}^{\infty} \mathcal{L}_{n}(k)
$$

be the free graded Lie algebra generated by $H_{n}$. Also let $\mathcal{L}_{n}^{\mathbb{Q}}=\mathcal{L}_{n} \otimes \mathbb{Q}$. We set

$$
\operatorname{Der}^{+}\left(\mathcal{L}_{n}\right)=\left\{\text { derivation } D \text { of } \mathcal{L}_{n} \text { with positive degree }\right\}
$$

which has a natural structure of a graded Lie algebra over $\mathbb{Z}$. The degree $k$ part of this graded Lie algebra can be expressed as

$$
\operatorname{Der}^{+}\left(\mathcal{L}_{n}\right)(k)=\operatorname{Hom}\left(H_{n}, \mathcal{L}_{n}(k+1)\right)
$$

and we have

$$
\operatorname{Der}^{+}\left(\mathcal{L}_{n}\right)=\bigoplus_{k=1}^{\infty} \operatorname{Der}^{+}\left(\mathcal{L}_{n}\right)(k)
$$

Similarly we consider $\operatorname{Der}^{+}\left(\mathcal{L}_{n}^{\mathbb{Q}}\right)$ which is a graded Lie algebra over $\mathbb{Q}$.

In the case where we are given an identification $\pi_{1}\left(\Sigma_{g} \backslash \operatorname{Int} D\right) \cong F_{2 g}$, we have the symplectic class $\omega_{0} \in \mathcal{L}_{2 g}(2)=\Lambda^{2} H_{2 g}$ and we can consider the following graded Lie subalgebra

$$
\begin{aligned}
\mathfrak{h}_{g, 1} & =\left\{D \in \operatorname{Der}^{+}\left(\mathcal{L}_{2 g}\right) ; D\left(\omega_{0}\right)=0\right\} \\
& =\bigoplus_{k=1}^{\infty} \mathfrak{h}_{g, 1}(k) .
\end{aligned}
$$

Similarly we have $\mathfrak{h}_{g, 1}^{\mathbb{Q}}=\mathfrak{h}_{g, 1} \otimes \mathbb{Q}$.

In our paper [76], for each $k$, we defined a certain homomorphism

$$
\operatorname{trace}(k): \operatorname{Der}^{+}\left(\mathcal{L}_{n}\right)(k) \longrightarrow S^{k} H_{n}
$$

where $S^{k} H_{n}$ denotes the $k$-th symmetric power of $H_{n}$. We call this "trace" because it is defined as the usual trace of the abelianized noncommutative Jacobian matrix of each homogeneous derivation. Here we recall the definition briefly from the above cited paper. Choose a basis $x_{1}, \cdots, x_{n}$ of $H_{n}=H_{1}\left(F_{n} ; \mathbb{Z}\right)$. We can consider $\mathcal{L}_{n}(k+1)$ as a natural submodule of $H_{n}^{\otimes(k+1)}$ consisting of all the Lie polynomials of degree $k+1$. For example $\left[x_{1}, x_{2}\right] \in \mathcal{L}_{n}(2)$ corresponds to the element $x_{1} \otimes x_{2}-x_{2} \otimes x_{1} \in H^{\otimes 2}$. By using the concept of the Fox free differential, 
we can also embed $\mathcal{L}_{n}(k+1)$ into the set $\left(H_{n}^{\otimes k}\right)^{n}$ of all the $n$-dimensional column vectors with entries in $H_{n}^{\otimes k}$ by the following correspondence

$$
\mathcal{L}_{n}(k+1) \ni \eta \longmapsto\left(\frac{\partial \eta}{\partial x_{i}}\right) \in\left(H_{n}^{\otimes k}\right)^{n} .
$$

Here for each monomial $\eta \in \mathcal{L}_{n}(k+1) \subset H_{n}^{\otimes(k+1)}$ which is uniquely expressed as

$$
\eta=\eta_{1} \otimes x_{1}+\cdots+\eta_{n} \otimes x_{n} \quad\left(\eta_{i} \in H_{n}^{\otimes k}\right),
$$

we have

$$
\frac{\partial \eta}{\partial x_{i}}=\eta_{i}
$$

Definition 21. In the above terminologies, the $k$-th trace $\operatorname{trace}(k)$ : $\operatorname{Der}^{+}\left(\mathcal{L}_{n}\right)(k) \longrightarrow S^{k} H_{n}$ is defined by

$$
\operatorname{trace}(k)(f)=\left(\sum_{i=1}^{n} \frac{\partial f\left(x_{i}\right)}{\partial x_{i}}\right)^{\mathrm{ab}}
$$

where $f \in \operatorname{Der}^{+}\left(\mathcal{L}_{n}\right)(k)=\operatorname{Hom}\left(H_{n}, \mathcal{L}_{n}(k+1)\right)$ and the superscript ab denotes the natural projection $H_{n}^{\otimes k} \rightarrow S^{k} H_{n}$.

Remark 22. If we identify the target $\operatorname{Hom}\left(H_{n}, \mathcal{L}_{n}(k+1)\right)$ of $\operatorname{trace}(k)$ with

$$
H_{n}^{*} \otimes \mathcal{L}_{n}(k+1) \subset H_{n}^{*} \otimes H_{n}^{\otimes(k+1)}
$$

where $H_{n}^{*}=\operatorname{Hom}\left(H_{n}, \mathbb{Z}\right)$ denotes the dual space of $H_{n}$, then it follows immediately from the definition that $\operatorname{trace}(k)$ is equal to the restriction of the contraction

$$
C_{k+1}: H_{n}^{*} \otimes H_{n}^{\otimes(k+1)} \longrightarrow H_{n}^{\otimes k}
$$

followed by the abelianization $H_{n}^{\otimes k} \rightarrow S^{k} H_{n}$. Here

$$
C_{k+1}\left(f \otimes u_{1} \otimes \cdots \otimes u_{k+1}\right)=f\left(u_{k+1}\right) u_{1} \otimes \cdots \otimes u_{k}
$$

for $f \in H_{n}^{*}, u_{i} \in H_{n}$. Also it is easy to see that, if we replace $C_{k+1}$ with $C_{1}$ defined by

$$
C_{1}\left(f \otimes u_{1} \otimes \cdots \otimes u_{k+1}\right)=f\left(u_{1}\right) u_{2} \otimes \cdots \otimes u_{k+1}
$$

in the above discussion, then we obtain $(-1)^{k} \operatorname{trace}(k)$.

Example 23. Let $\operatorname{ad}_{x_{2}}\left(x_{1}\right)^{k} \in \operatorname{Der}^{+}\left(\mathcal{L}_{n}\right)(k)$ be the element defined by

$$
\begin{aligned}
& \operatorname{ad}_{x_{2}}\left(x_{1}\right)^{k}\left(x_{2}\right)=\left[x_{1},\left[x_{1},\left[\cdots,\left[x_{1}, x_{2}\right] \cdots\right] \quad\left(k \text {-times } x_{1}\right)\right.\right. \\
& \operatorname{ad}_{x_{2}}\left(x_{1}\right)^{k}\left(x_{i}\right)=0 \quad(i \neq 2) .
\end{aligned}
$$

Then a direct computation shows that

$$
\operatorname{trace}(k)\left(\operatorname{ad}_{x_{2}}\left(x_{1}\right)^{k}\right)=x_{1}^{k} .
$$


As was mentioned in [76], the traces are $G L\left(H_{n}\right)$-equivariant in an obvious sense. Since $x_{1}^{k}$ generates $S^{k} H_{n}$ as a $G L\left(H_{n}\right)$-module, the above example implies that the mapping trace $(k): \operatorname{Der}^{+}\left(\mathcal{L}_{n}\right)(k) \longrightarrow S^{k} H_{n}$ is surjective for any $k$. Another very important property of the traces proved in the above cited paper is that they vanish identically on the commutator ideal $\left[\operatorname{Der}^{+}\left(\mathcal{L}_{n}\right), \operatorname{Der}^{+}\left(\mathcal{L}_{n}\right)\right]$. Hence we have the following surjective homomorphism of graded Lie algebras

$$
\left(\tau_{1}, \oplus_{k} \operatorname{trace}(k)\right): \operatorname{Der}^{+}\left(\mathcal{L}_{n}\right) \longrightarrow \operatorname{Hom}\left(H_{n}, \Lambda^{2} H_{n}\right) \oplus \bigoplus_{k=2}^{\infty} S^{k} H_{n}
$$

where the target is understood to be an abelian Lie algebra. We have also proved that, for any $k$, trace $(2 k)$ vanishes identically on $\mathfrak{h}_{g, 1}$ and that trace $(2 k+1): \mathfrak{h}_{g, 1}^{\mathbb{Q}}(2 k+1) \rightarrow S^{2 k+1} H_{2 g}^{\mathbb{Q}}$ is surjective. Thus we have a surjective homomorphism

$$
\left(\tau_{1}, \oplus_{k} \operatorname{trace}(2 k+1)\right): \mathfrak{h}_{g, 1}^{\mathbb{Q}} \longrightarrow \Lambda^{3} H_{2 g}^{\mathbb{Q}} \oplus \bigoplus_{k=1}^{\infty} S^{2 k+1} H_{2 g}^{\mathbb{Q}}
$$

of graded Lie algebras which we conjectured to give the abelianization of the Lie algebra $\mathfrak{h}_{g, 1}^{\mathbb{Q}}$ (see Conjecture 6.10 of [80]).

Recently Kassabov 47. (Theorem 1.4.11) proved the following remarkable result. Let $x_{1}, \cdots, x_{n}$ be a basis of $H_{n}$ as before.

Theorem 24 (Kassabov). Up to degree $n(n-1)$, the graded Lie algebra $\operatorname{Der}^{+}\left(\mathcal{L}_{n}^{\mathbb{Q}}\right)$ is generated as a Lie algebra and $\mathfrak{s l}(n, \mathbb{Q})$-module by the elements $\operatorname{ad}\left(x_{1}\right)^{k}(k=1,2, \cdots)$ and the element $D$ which sends $x_{1}$ to $\left[x_{2}, x_{3}\right]$ and $x_{i}(i \neq 1)$ to 0 .

If we combine this theorem with the concept of the traces, we obtain the following.

Theorem 25. The surjective Lie algebra homomorphism

$$
\left(\tau_{1}, \oplus_{k} \operatorname{trace}(k)\right): \operatorname{Der}^{+}\left(\mathcal{L}_{n}^{\mathbb{Q}}\right) \longrightarrow \operatorname{Hom}\left(H_{n}^{\mathbb{Q}}, \Lambda^{2} H_{n}^{\mathbb{Q}}\right) \oplus \bigoplus_{k=2}^{\infty} S^{k} H_{n}^{\mathbb{Q}},
$$

induced by the degree 1 part and the traces, gives the abelianization of the graded Lie algebra $\operatorname{Der}^{+}\left(\mathcal{L}_{n}^{\mathbb{Q}}\right)$ up to degree $n(n-1)$ so that any element of degree $2 \leq d \leq n(n-1)$ with vanishing trace belongs to the commutator ideal $\left[\operatorname{Der}^{+}\left(\mathcal{L}_{n}^{\mathbb{Q}}\right), \operatorname{Der}^{+}\left(\mathcal{L}_{n}^{\mathbb{Q}}\right)\right]$. Furthermore, any $\mathfrak{s l}(n, \mathbb{Q})$ equivariant splitting to this abelianization generates $\operatorname{Der}^{+}\left(\mathcal{L}_{n}^{\mathbb{Q}}\right)$ in this range. Hence stably there exists an isomorphism

$$
H_{1}\left(\operatorname{Der}^{+}\left(\mathcal{L}_{\infty}^{\mathbb{Q}}\right)\right) \cong \operatorname{Hom}\left(H_{\infty}^{\mathbb{Q}}, \Lambda^{2} H_{\infty}^{\mathbb{Q}}\right) \oplus \bigoplus_{k=2}^{\infty} S^{k} H_{\infty}^{\mathbb{Q}}
$$


and the degree 1 part and (any $\mathfrak{s l}(n, \mathbb{Q})$-equivariant splittings of) the traces generate $\operatorname{Der}^{+}\left(\mathcal{L}_{\infty}^{\mathbb{Q}}\right)$.

Although the structure of $\mathfrak{h}_{g, 1}^{\mathbb{Q}}$ is much more complicated than that of $\operatorname{Der}^{+}\left(\mathcal{L}_{n}^{\mathbb{Q}}\right)$, Kassabov's argument adapted to this case together with some additional idea will produce enough information about the generation as well as the abelianization of $\mathfrak{h}_{g, 1}^{\mathbb{Q}}$ in a certain stable range. Details will be given in our forthcoming paper 83 . It follows that any element in the Lie algebra $\mathfrak{h}_{\infty, 1}^{\mathbb{Q}}$ can be expressed in terms of the degree 1 part and the traces.

\section{The SECOND COHOMOLOGY of $\mathfrak{h}_{g, 1}^{\mathbb{Q}}$}

In this section, we define a series of elements in $H^{2}\left(\mathfrak{h}_{g, 1}^{\mathbb{Q}}\right)^{S p}$ which denotes the $S p$-invariant part of the second cohomology of the graded Lie algebra $\mathfrak{h}_{g, 1}^{\mathbb{Q}}$.

As is well known, $U_{\mathbb{Q}}=\Lambda^{3} H_{\mathbb{Q}} / \omega_{0} \wedge H_{\mathbb{Q}}$ and $S^{2 k+1} H_{\mathbb{Q}}(k=1,2, \cdots)$ are all irreducible representations of $\operatorname{Sp}(2 g, \mathbb{Q})$. It is well known in the representation theory that, for any irreducible representation $V$ of the algebraic group $\operatorname{Sp}(2 g, \mathbb{Q})$, the tensor product $V \otimes V$ contains a unique trivial summand $\mathbb{Q} \subset V \otimes V$ (cf. [20] for generalities of the representations of the algebraic group $\operatorname{Sp}(2 g, \mathbb{Q})$ ). In our case where $V$ is any of the above irreducible representations, it is easy to see that the trivial summand appears in the second exterior power part $\Lambda^{2} V \subset$ $V \otimes V$. It follows that each of

$$
\Lambda^{2} U_{\mathbb{Q}}, \Lambda^{2} S^{3} H_{\mathbb{Q}}, \Lambda^{2} S^{5} H_{\mathbb{Q}}, \cdots
$$

contains a unique trivial summand $\mathbb{Q}$. Let

$$
\iota_{1}: \Lambda^{2} U_{\mathbb{Q}} \rightarrow \mathbb{Q}, \quad \iota_{2 k+1}: \Lambda^{2} S^{2 k+1} H_{\mathbb{Q}} \rightarrow \mathbb{Q} \quad(k=1,2, \cdots)
$$

be the unique (up to scalars) $S p$-equivariant homomorphism. We would like to call them higher intersection pairing on surfaces which generalize the usual one $\Lambda^{2} H_{\mathbb{Q}} \rightarrow \mathbb{Q}$. We can write

$$
\iota_{1} \in H^{2}\left(U_{\mathbb{Q}}\right)^{S p}, \quad \iota_{2 k+1} \in H^{2}\left(S^{2 k+1} H_{\mathbb{Q}}\right)^{S p} .
$$

Definition 26. We define the cohomology classes

$$
e_{1}, t_{3}, t_{5}, \cdots \in H^{2}\left(\mathfrak{h}_{g, 1}^{\mathbb{Q}}\right)^{S p}
$$

by setting

$$
e_{1}=\bar{\tau}_{1}^{*}\left(\iota_{1}\right), \quad t_{2 k+1}=\operatorname{trace}(2 k+1)^{*}\left(\iota_{2 k+1}\right)
$$

where $\bar{\tau}_{1}$ denotes the composition $\mathfrak{h}_{g, 1}^{\mathbb{Q}} \rightarrow \Lambda^{3} H_{\mathbb{Q}} \rightarrow U_{\mathbb{Q}}$. 
Conjecture 27. The classes $e_{1}, t_{3}, t_{5}, \cdots$ are all non-trivial. Furthermore they are linearly independent and form a basis of $H^{2}\left(\mathfrak{h}_{g, 1}^{\mathbb{Q}}\right)^{S p}$.

Remark 28. The element $e_{1}$ is the Lie algebra version of the first Mumford-Morita-Miller class (we use the same notation).

Remark 29. The Lie algebra $\mathfrak{h}_{g, 1}^{\mathbb{Q}}$ is graded so that the cohomology group $H^{2}\left(\mathfrak{h}_{g, 1}^{\mathbb{Q}}\right)^{S p}$ is bigraded. Let $H^{2}\left(\mathfrak{h}_{g, 1}^{\mathbb{Q}}\right)_{n}^{S p}$ denote the weight $n$ part of $H^{2}\left(\mathfrak{h}_{g, 1}^{\mathbb{Q}}\right)^{S p}$ (see $\S 9$ for more details). Then by definition we have

$$
e_{1} \in H^{2}\left(\mathfrak{h}_{g, 1}^{\mathbb{Q}}\right)_{2}^{S p}, \quad t_{2 k+1} \in H^{2}\left(\mathfrak{h}_{g, 1}^{\mathbb{Q}}\right)_{4 k+2}^{S p} .
$$

Hence if the elements $e_{1}, t_{3}, t_{5}, \cdots$ are non-trivial, then they are automatically linearly independent. Thus the above conjecture can be rewritten as

$$
H^{2}\left(\mathfrak{h}_{g, 1}^{\mathbb{Q}}\right)_{n}^{S p} \cong \begin{cases}\mathbb{Q} & (n=2,6,10,14, \cdots) \\ 0 & \text { (otherwise) }\end{cases}
$$

where the summands $\mathbb{Q}$ in degrees $2,6,10, \cdots$ are generated by the above classes.

As for the non-triviality, all we know at present is the non-triviality of $e_{1}, t_{3}, t_{5}$. The non-triviality of the class $t_{2 k+1}$ is the same as that of the class $\mu_{k}$ because of the theorem of Kontsevich described in $\S 9$. This will be explained in that section.

\section{Constructing COHOmology Classes of $\mathfrak{h}_{g, 1}^{\mathbb{Q}}$}

In this section, we describe a general method of constructing $S p$ invariant cohomology classes of the Lie algebra $\mathfrak{h}_{g, 1}^{\mathbb{Q}}$ which generalize the procedure given in the previous section. As was already mentioned in our paper 80, the homomorphism (111) induces the following homomorphim in the $S p$-invariant part of the cohomology

$$
H^{*}\left(\Lambda^{3} H_{\mathbb{Q}} \oplus \bigoplus_{k=1}^{\infty} S^{2 k+1} H_{2 g}^{\mathbb{Q}}\right)^{S p} \longrightarrow H^{*}\left(\mathfrak{h}_{g, 1}^{\mathbb{Q}}\right)^{S p} .
$$

By the same way as in 78, 51, the left hand side can be computed by certain polynomial algebra

$$
\mathbb{Q}\left[\Gamma ; \Gamma \in \mathcal{G}^{\text {odd }}\right]
$$

generated by graphs belonging to $\mathcal{G}^{\text {odd }}$ which denotes the set of all isomorphism classes of connected graphs with valencies in the set

$$
3,3,5,7, \cdots
$$


of odd integers. Here we write two copies of 3 because of different roles: the first one is related to the target $\Lambda^{3} H_{\mathbb{Q}}$ of $\tau_{1}$ (alternating) while the second one is related to the target $S^{3} H_{\mathbb{Q}}$ of trace(3) (symmetric). The other $2 k+1(k=2,3, \cdots)$ are related to the target $S^{2 k+1} H_{\mathbb{Q}}$ of trace $(2 k+1)$. Thus we obtain a homomorphism

$$
\Phi: \mathbb{Q}\left[\Gamma ; \Gamma \in \mathcal{G}^{\text {odd }}\right] \longrightarrow H^{*}\left(\mathfrak{h}_{g, 1}^{\mathbb{Q}}\right)^{S p} .
$$

The elements $e_{1}, t_{3}, t_{5}, \cdots$ defined in Definition 26 arise as the images, under $\Phi$, of those graphs with exactly two vertices which are connected by $3,3,5,7, \cdots$ edges.

Remark 30. As was mentioned already in the previous section $\S 6$, the cohomology of $\mathfrak{h}_{g, 1}^{\mathbb{Q}}$ is bigraded. Let $\Gamma \in \mathcal{G}^{\text {odd }}$ be a connected graph whose valencies are $v_{3}^{a}$ times 3 (alternating), $v_{3}^{s}$ times 3 (symmetric) and $v_{2 k+1}$ times $2 k+1(2 k+1>3)$. Then

$$
\Phi(\Gamma) \in H^{d}\left(\mathfrak{h}_{g, 1}^{\mathbb{Q}}\right)_{n}^{S p}
$$

where

$$
\begin{aligned}
& d=v_{3}^{a}+v_{3}^{s}+v_{5}+v_{7}+\cdots \\
& n=v_{3}^{a}+3 v_{3}^{s}+5 v_{5}+7 v_{7}+\cdots .
\end{aligned}
$$

Observe that $n+2 v_{3}^{a}$ is equal to twice of the number of edges of $\Gamma$. It follows that $n$ (and hence $d$ ) is always an even integer.

Problem 31. Find explicit graphs $\Gamma \in \mathcal{G}^{\text {odd }}$ such that the corresponding homology classes $\Phi(\Gamma)$ are non-trivial.

\section{THREE GROUPS BEYOND THE MAPPING CLASS GROUP}

In view of the definition of the Lie algebra $\mathfrak{h}_{g, 1}$ (see $\S 5$ ), we may say that it is the "Lie algebra version" of the mapping class group $\mathcal{M}_{g, 1}$. However the result of the author [76] showed that it is too big to be considered so and the following question arose: what is the algebraic and/or geometric meaning of the complement of the image of $\mathcal{M}_{g, 1}$ in $\mathfrak{h}_{g, 1}$ ? Two groups came into play in this framework in the 1990's. One is the arithmetic mapping class group through the works of number theorists, notably Oda, Nakamura and Matsumoto, and the other is Out $F_{n}$ via the theorem of Kontsevich described in the next section $\S 9$. In this section, we would like to consider the former group briefly from a very limited point of view (see [87] 66 and references therein 
for details). The latter group was already introduced in $\S 4$ and will be further discussed in $\S 10$.

More recently, it turned out that we have to treat one more group in the above setting and that is the group of homology cobordism classes of homology cylinders. This will be discussed in $\S 11$ below. We strongly expect that the traces will give rise to meaningful invariants in each of these three groups beyond the mapping classs group.

Now we consider the first group above. The action of $\mathcal{M}_{g, 1}$ on the lower central series of $\Gamma=\pi_{1}\left(\Sigma_{g} \backslash \operatorname{Int} D\right)$ induces a filtration $\left\{\mathcal{M}_{g, 1}(k)\right\}_{k}$ on $\mathcal{M}_{g, 1}$ as follows. Let $\Gamma_{1}=[\Gamma, \Gamma]$ be the commutator subgroup of $\Gamma$ and inductively define $\Gamma_{k+1}=\left[\Gamma, \Gamma_{k}\right](k=1,2, \cdots)$. The quotient group $N_{k}=\Gamma / \Gamma_{k}$ is called the $k$-th nilpotent quotient of $\Gamma$. Note that $N_{1}$ is canonically isomorphic to $H_{2 g}=H_{1}\left(\Sigma_{g} ; \mathbb{Z}\right)$. Now we set

$$
\mathcal{M}_{g, 1}(k)=\left\{\varphi \in \mathcal{M}_{g, 1} ; \varphi \text { acts on } N_{k} \text { trivially }\right\} .
$$

Thus the first group $\mathcal{M}_{g, 1}(1)$ in this filtration is nothing but the Torelli group $\mathcal{I}_{g, 1}$. As is well known, the quotient group $\Gamma_{k} / \Gamma_{k+1}$ can be identified with the $(k+1)$-st term $\mathcal{L}_{2 g}(k+1)$ of the free graded Lie algebra generated by $H_{2 g}$ (see $\S 5$ ). It can be checked that the correspondence

$$
\begin{aligned}
& \mathcal{M}_{g, 1}(k) \ni \varphi \longmapsto \\
& \Gamma \ni \alpha \mapsto \varphi_{*}(\alpha) \alpha^{-1} \in \Gamma_{k} / \Gamma_{k+1} \cong \mathcal{L}_{2 g}(k+1)
\end{aligned}
$$

descends to a homomorphism

$$
\tau_{k}: \mathcal{M}_{g, 1}(k) \longrightarrow \operatorname{Hom}\left(H_{2 g}, \mathcal{L}_{2 g}(k+1)\right)
$$

which is now called the $k$-th Johnson homomorphism because it was introduced by Johnson (see [40]41]). Furthermore it turns out that the totality $\left\{\tau_{k}\right\}_{k}$ of these homomorphims induces an injective homomorphism of graded Lie algebras

$$
\operatorname{Gr}^{+}\left(\mathcal{M}_{g, 1}\right)=\bigoplus_{k=1}^{\infty} \mathcal{M}_{g, 1}(k) / \mathcal{M}_{g, 1}(k+1) \longrightarrow \mathfrak{h}_{g, 1}
$$

(see [76] 80] for details). Although there have been obtained many important results concerning the image of the above homomorphism, the following is still open.

Problem 32. Determine the image as well as the cokernel of the homomorphism (15) explicitly.

Note that Hain [29] proved that the image of (15), after tensored with $\mathbb{Q}$, is precisely the Lie subalgebra generated by the degree 1 part. However it is unclear which part of $\mathfrak{h}_{g, 1}^{\mathbb{Q}}$ belongs to this Lie subalgebra. 
In relation to this problem, Oda predicted, in the late 1980's, that there should arise "arithmetic obstructions" to the surjectivity of Johnson homomorphism. More precisely, based on the theory of Ihara in number theory which treated mainly the case $g=0, n=3$, he expected that the absolute Galois group $\operatorname{Gal}(\overline{\mathbb{Q}} / \mathbb{Q})$ should "appear" in $\mathfrak{h}_{g, 1} \otimes \mathbb{Z}_{\ell}$ outside of the geometric part and which should be $S p$-invariant for any genus $g$ and for any prime $\ell$. In 1994, Nakamura 86 proved, among other results, that this is in fact the case (see also Matsumoto 65]). This was the second obstruction to the surjectivity of Johnson homomorphism, the first one being the traces in [76]. This raised the following problem.

Problem 33. Describe the Galois images in $\mathfrak{h}_{g, 1} \otimes \mathbb{Z}_{\ell}$.

The above result was proved by analyzing the number theoretical enhancement of the Johnson homomorphism where the geometric mapping class group is replaced by the arithmetic mapping class group which is expressed as an extension

$$
1 \longrightarrow \hat{\mathcal{M}}_{g}^{n} \longrightarrow \pi_{1}^{a l g} \mathbf{M}_{g}^{n} / \mathbb{Q} \longrightarrow \operatorname{Gal}(\overline{\mathbb{Q}} / \mathbb{Q}) \longrightarrow 1
$$

and studied by Grothendieck, Deligne, Ihara, Drinfel'd and many number theorists. Nakamura continued to study the structure of the mapping class group from the point of view of number theory extensively (see e.g. 88 [89]). On the other hand, Hain and Matsumoto recently proved remarkable results concerning this subject (see 31] 32]). In view of deep theories in number theory, as well as the above explicit results, it seems to be conjectured that there should exist an embedding

$$
\text { FreeLie }_{\mathbb{Z}}\left(\sigma_{3}, \sigma_{5}, \cdots\right) \subset \mathfrak{h}_{g, 1}
$$

of certain free graded Lie algebra over $\mathbb{Z}$ generated by certain elements $\sigma_{3}, \sigma_{5}, \cdots$, corresponding to the Soulé elements, into $\mathfrak{h}_{g, 1}$ such that the tensor product of it with $\mathbb{Z}_{\ell}$ coincides with the image of $\operatorname{Gal}(\overline{\mathbb{Q}} / \mathbb{Q})$ for any prime $\ell$.

We expect that the above conjectured free graded Lie algebra (the motivic Lie algebra) can be realized inside $\mathfrak{h}_{g, 1}$ (in fact inside the commutator ideal $\left[\mathfrak{h}_{g, 1}, \mathfrak{h}_{g, 1}\right]$ ) explicitly in terms of the traces. In some sense, the elements $\sigma_{2 k+1}$ should be decomposable in higher genera. Here we omit the precise form of the expected formula which will be given in a forthcoming paper.

Finally we mention the analogue of the Johnson homomorphisms for the group Aut $F_{n}$ very briefly. Prior to the work of Johnson, Andreadakis [1] introduced and studied the filtration on $\left\{\text { Aut } F_{n}(k)\right\}_{k}$ which is induced from the action of Aut $F_{n}$ on the lower central series 
of $F_{n}$. The first group Aut $F_{n}(1)$ in this filtration is nothing but the group IAut $_{n}$. It can be checked that an analogous procedure as in the case of the mapping class group gives rise to certain homomorphisms

$$
\tau_{k}: \operatorname{Aut} F_{n}(k) \longrightarrow \operatorname{Hom}\left(H_{n}, \mathcal{L}_{n}(k+1)\right)
$$

and the totality $\left\{\tau_{k}\right\}_{k}$ of these homomorphims induces an injective homomorphism of graded Lie algebras

$$
\operatorname{Gr}^{+}\left(\operatorname{Aut} F_{n}\right)=\bigoplus_{k=1}^{\infty} \operatorname{Aut} F_{n}(k) / \operatorname{Aut} F_{n}(k+1) \longrightarrow \operatorname{Der}^{+}\left(\mathcal{L}_{n}\right)
$$

We refer to [49] 93] 98] for some of the recent works related to the above homomorphism.

\section{A theOREM OF Kontsevich}

In this section, we recall a theorem of Kontsevich described in [55] [56] which is the key result for the argument given in the next section. See also the paper 9] by Conant and Vogtmann for a detailed proof as well as discussion of this theorem in the context of cyclic operads. In the above cited papers, Kontsevich considered three kinds of infinite dimensional Lie algebras denoted by $\mathfrak{c}_{g}, \mathfrak{a}_{g}, \mathfrak{l}_{g}$ (commutative, associative, and lie version, respectively). The latter two Lie algebras are defined by

$$
\begin{array}{r}
\mathfrak{a}_{g}=\left\{\text { derivation } D \text { of the tensor algebra } T^{*}\left(H_{\mathbb{Q}}\right)\right. \\
\text { such that } \left.D\left(\omega_{0}\right)=0\right\} \\
\mathfrak{l}_{g}=\left\{\text { derivation } D \text { of the free Lie algebra } \mathcal{L}_{2 g}^{\mathbb{Q}} \subset T^{*}\left(H_{\mathbb{Q}}\right)\right. \\
\text { such that } \left.D\left(\omega_{0}\right)=0\right\} .
\end{array}
$$

There is a natural injective Lie algebra homomorphism $\mathfrak{l}_{g} \rightarrow \mathfrak{a}_{g}$. The degree 0 part of both of $\mathfrak{a}_{g}, \mathfrak{l}_{g}$ is the Lie algebra $\mathfrak{s p}(2 g, \mathbb{Q})$ of $\operatorname{Sp}(2 g, \mathbb{Q})$. Let $\mathfrak{a}_{g}^{+}$(resp. $\mathfrak{l}_{g}^{+}$) denote the Lie subalgebra of $\mathfrak{a}_{g}$ (resp. $\mathfrak{l}_{g}$ ) consisting of derivations with positive degrees. Then the latter one $\mathfrak{l}_{g}^{+}$is nothing other than the Lie algebra $\mathfrak{h}_{g, 1}^{\mathbb{Q}}$ considered in $\S 5$. Now Kontsevich described the stable homology groups of the above three Lie algebras (where $g$ tends to $\infty$ ) in terms of cohomology groups of graph complexes, moduli spaces $\mathbf{M}_{g}^{m}$ of Riemann surfaces and the outer automorphism groups Out $F_{n}$ of free groups (or the moduli space of graphs), respectively. Here is the statement for the cases of $\mathfrak{a}_{\infty}, \mathfrak{l}_{\infty}$. 
Theorem 34 (Kontsevich). There are isomorphisms

$$
\begin{aligned}
& P H_{*}\left(\mathfrak{a}_{\infty}\right) \cong P H_{*}(\mathfrak{s p}(\infty, \mathbb{Q})) \oplus \bigoplus_{g \geq 0, m \geq 1,2 g-2+m>0} H^{*}\left(\mathbf{M}_{g}^{m} ; \mathbb{Q}\right)^{\mathfrak{S}_{m}}, \\
& P H_{*}\left(\mathfrak{l}_{\infty}\right) \cong P H_{*}(\mathfrak{s p}(\infty, \mathbb{Q})) \oplus \bigoplus_{n \geq 2} H^{*}\left(\text { Out } F_{n} ; \mathbb{Q}\right) .
\end{aligned}
$$

Here $P$ denotes the primitive parts of $H_{*}\left(\mathfrak{a}_{\infty}\right), H_{*}\left(\mathfrak{l}_{\infty}\right)$ which have natural structures of Hopf algebras and $\mathbf{M}_{g}^{m}$ denotes the moduli space of genus $g$ smooth curves with $m$ punctures.

Here is a very short outline of the proof of the above theorem. Using natural cell structure of the Riemann moduli space $\mathbf{M}_{g}^{m}(m \geq 1)$ and the moduli space $\mathbf{G}_{n}$ of graphs, which serves as the (rational) classifying space of Out $F_{n}$ by [11], Kontsevich introduced a natural filtration on the cellular cochain complex of these moduli spaces. Then he proved that the associated spectral sequence degenerates at the $E_{2}$-term and only the diagonal terms remain to be non-trivial. On the other hand, by making use of classical representation theory for the group $\operatorname{Sp}(2 g, \mathbb{Q})$, he constructed a quasi isomorphism between the $E_{1}$-term and the chain complexes of the relevant Lie algebras $\left(\mathfrak{a}_{\infty}\right.$ or $\left.\mathfrak{l}_{\infty}\right)$. For details, see the original papers cited above as well as [9].

There is also the dual version of the above theorem which connects the primitive cohomology of the relevant Lie algebras with the homology groups of the moduli space or Out $F_{n}$. We would like to describe it in a detailed form because this version is most suitable for our purpose. The Lie algebras $\mathfrak{l}_{\infty}^{+}, \mathfrak{a}_{\infty}^{+}$are graded. Hence their $S p$-invariant cohomology groups are bigraded. Let $H^{k}\left(\mathfrak{l}_{\infty}^{+}\right)_{n}^{S p}$ and $H^{k}\left(\mathfrak{a}_{\infty}^{+}\right)_{n}^{S p}$ denote the weight $n$ part of $H^{k}\left(\mathfrak{l}_{\infty}^{+}\right)^{S p}$ and $H^{k}\left(\mathfrak{a}_{\infty}^{+}\right)^{S p}$ respectively. Then we have the following isomorphisms.

$$
\begin{aligned}
& P H^{k}\left(\mathfrak{l}_{\infty}^{+}\right)_{2 n}^{S p} \cong H_{2 n-k}\left(\text { Out } F_{n+1} ; \mathbb{Q}\right) \\
& P H^{k}\left(\mathfrak{a}_{\infty}^{+}\right)_{2 n}^{S p} \cong \bigoplus_{2 g-2+m=n} H_{2 n-k}\left(\mathbf{M}_{g}^{m} ; \mathbb{Q}\right)_{\mathfrak{S}_{m}}
\end{aligned}
$$

\section{Constructing homology Classes of Out $F_{n}$}

In this section, we combine our construction of many cohomology classes in $H^{*}\left(\mathfrak{h}_{g, 1}^{\mathbb{Q}}\right)^{S p}$ given in $\S 6, \S 7$ with Kontsevich's theorem given in $\S 9$ to produce homology classes of the group Out $F_{n}$. 
First we see that the homomorphism given in (13) is stable with respect to $g$. More precisely, the following diagram is commutative

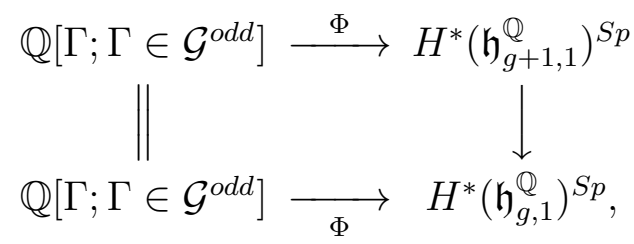

where the right vertical map is induced by the inclusion $\mathfrak{h}_{g, 1} \rightarrow \mathfrak{h}_{g+1,1}$. This follows from the fact that the traces trace $(2 k+1)$ as well as $\tau_{1}$ are all stable with respect to $g$ in an obvious way.

Next, we see that the cohomology class $\Phi(\Gamma) \in H^{*}\left(\mathfrak{h}_{\infty, 1}^{\mathbb{Q}}\right)^{S p}$ obtained in this way is primitive if and only if $\Gamma$ is connected. Keeping in mind the fact $\mathfrak{h}_{\infty, 1}^{\mathbb{Q}}=\mathfrak{l}_{\infty}^{+}$, the property (14), as well as the version of Kontsevich's theorem given in (17) we now obtain the following theorem.

Theorem 35. Associated to each connected graph $\Gamma \in \mathcal{G}^{\text {odd }}$ whose valencies are $v_{3}^{a}$ times 3 (alternating), $v_{3}^{s}$ times 3 (symmetric) and $v_{2 k+1}$ times $2 k+1(2 k+1>3)$, we have a homology class

$$
\Phi(\Gamma) \in H_{2 n-d}\left(\text { Out } F_{n+1} ; \mathbb{Q}\right)
$$

where

$$
d=v_{3}^{a}+v_{3}^{s}+v_{5}+v_{7}+\cdots, 2 n=v_{3}^{a}+3 v_{3}^{s}+5 v_{5}+7 v_{7}+\cdots .
$$

Remark 36. Let $\Gamma_{2 k+1}$ be the connected graph with two vertices both of which have valency $2 k+1$. Then $d=2,2 n=4 k+2$ and $\Phi\left(\Gamma_{2 k+1}\right) \in$ $H_{4 k}\left(\right.$ Out $\left.F_{2 k+2} ; \mathbb{Q}\right)$ is the class $\mu_{k}$ already mentioned in $\S 4$.

The following problem is an enhancement of Problem 31 in the context of the homology of the moduli space of graphs rather than the cohomology of the Lie algebra $\mathfrak{h}_{g, 1}^{\mathbb{Q}}$.

Problem 37. Give examples of odd valent graphs $\Gamma$ whose associated homology classes $\Phi(\Gamma) \in H_{*}\left(\right.$ Out $\left.F_{n} ; \mathbb{Q}\right)$ are non-trivial as many as possible. Also compare these classes with the homology classes constructed by Conant and Vogtmann [10] as explicit cycles in the moduli space of graphs. Furthermore investigate whether these classes survive in $H_{*}(\mathrm{GL}(n, \mathbb{Z}) ; \mathbb{Q})$, or else come from $H_{*}\left(\mathrm{IOut}_{n} ; \mathbb{Q}\right)$, or not.

Remark 38. It seems that the geometric meaning of Kontsevich's theorem is not very well understood yet. In particular, there is almost no known relations between the classes $\Phi(\Gamma) \in H_{*}\left(\right.$ Out $\left.F_{n} ; \mathbb{Q}\right)$ where the rank $n$ varies. However, there should be some unknown structures 
here. For example, there are graphs $\Gamma$ whose associated classes $\Phi(\Gamma)$ lie in $H_{8}\left(\right.$ Out $\left.F_{n} ; \mathbb{Q}\right)$ for $n=7,8$ which might be closely related to the class $\mu_{2} \in H_{8}\left(\right.$ Out $\left.F_{6} ; \mathbb{Q}\right)$.

\section{Group of homology COBORDISM ClASSES OF HOMOLOGY CYLINDERS}

In his theory developed in [27, Habiro introduced the concept of homology cobordism of surfaces and proposed interesting problems concerning it. Goussarov [26] also studied the same thing in his theory. It played an important role in the classification theory of 3-manifolds now called the Goussarov-Habiro theory. Later Garoufalidis and Levine 24] and Levine [60] used this concept to define a group $\mathcal{H}_{g, 1}$ which consists of homology cobordism classes of homology cylinders on $\Sigma_{g} \backslash \operatorname{Int} D$. We refer to the above papers for the definition (we use the terminology homology cylinder following them) as well as many interesting questions concerning the structure of $\mathcal{H}_{g, 1}$. It seems that the importance of this group is growing recently.

Here we summarize some of the results of [24 60] which will be necessary for our purpose here. Consider $\Gamma=\pi_{1}\left(\Sigma_{g} \backslash \operatorname{Int} D\right)$, which is isomorphic to $F_{2 g}$, and let $\left\{\Gamma_{k}\right\}_{k}$ be its lower central series as before. Note that $\Gamma$ contains a particular element $\gamma \in \Gamma$ which corresponds to the unique relation in $\pi_{1} \Sigma_{g}$. They define

$$
\operatorname{Aut}_{0}\left(\Gamma / \Gamma_{k}\right)=\left\{f \in \operatorname{Aut}\left(\Gamma / \Gamma_{k}\right)\right.
$$

$f$ lifts to an endomorphism of $\Gamma$ which fixes $\left.\gamma \bmod \Gamma_{k+1}\right\}$.

By making a crucial use of a theorem of Stallings [102, for each $k$ they obtain a homomorphism

$$
\sigma_{k}: \mathcal{H}_{g, 1} \longrightarrow \operatorname{Aut}_{0}\left(\Gamma / \Gamma_{k}\right) \text {. }
$$

The following theorem given in [24] is a basic result for the study of the structure of the group $\mathcal{H}_{g, 1}$.

Theorem 39 (Garoufalidis-Levine). The above homomorphism $\sigma_{k}$ is surjective for any $k$.

They use the homomorphisms $\left\{\sigma_{k}\right\}_{k}$ to define a certain filtration $\left\{\mathcal{H}_{g, 1}(k)\right\}_{k}$ of $\mathcal{H}_{g, 1}$ and show that the Johnson homomorphisms are defined also on this group. Furthermore they are surjective so that 
there is an isomorphism

$$
\operatorname{Gr}^{+}\left(\mathcal{H}_{g, 1}\right)=\bigoplus_{k=1}^{\infty} \mathcal{H}_{g, 1}(k) / \mathcal{H}_{g, 1}(k+1) \cong \mathfrak{h}_{g, 1} .
$$

They concluded from this fact that $\mathcal{H}_{g, 1}$ contains $\mathcal{M}_{g, 1}$ as a subgroup. The homomorphisms $\sigma_{k}$ fit together to define a homomorphism

$$
\sigma: \mathcal{H}_{g, 1} \longrightarrow \lim _{\longleftarrow} \operatorname{Aut}_{0}\left(\Gamma / \Gamma_{k}\right)
$$

They show that this homomorphism is not surjective by using the argument of Levine [59]. We mention a recent paper [96] of Sakasai for a related work. Also they point out that, although the restriction of $\sigma$ to $\mathcal{M}_{g, 1}$ is injective, $\sigma$ has a rather big kernel because $\operatorname{Ker} \sigma$ at least contains the group

$$
\Theta_{\mathbb{Z}}^{3}=\{\text { oriented homology 3-sphere }\} / \text { homology cobordism. }
$$

It is easy to see that $\Theta_{\mathbb{Z}}^{3}$ is contained in the center of $\mathcal{H}_{g, 1}$ so that we have a central extension

$$
0 \longrightarrow \Theta_{\mathbb{Z}}^{3} \longrightarrow \mathcal{H}_{g, 1} \longrightarrow \overline{\mathcal{H}}_{g, 1} \longrightarrow 1
$$

where $\overline{\mathcal{H}}_{g, 1}=\mathcal{H}_{g, 1} / \Theta_{\mathbb{Z}}^{3}$.

The group $\Theta_{\mathbb{Z}}^{3}$ is a very important abelian group in low dimensional topology. In [21, Furuta first proved that this group is an infinitely generated group by making use of gauge theory. See also the paper [16] by Fintushel and Stern for another proof. However, only a few additive invariants are known on this group at present besides the classical surjective homomorphism

$$
\mu: \Theta_{\mathbb{Z}}^{3} \longrightarrow \mathbb{Z} / 2
$$

defined by the Rokhlin invariant. One is a non-trivial homomorphism $\Theta_{\mathbb{Z}}^{3} \longrightarrow \mathbb{Z}$ constructed by Fr $\phi y$ shov [17] and the other is given by Ozsváth and Szabó 92 as an application of their Heegaard Floer homology theory. Neumann [90] and Siebenmann [101 defined an invariant $\bar{\mu}$ for plumbed homology 3-spheres and Saveliev [99] introduced his $\nu$ invariant for any homology sphere by making use of the Floer homology. On the other hand, Fukumoto and Furuta (see [18 [19]) defined certain invariants for plumbed homology 3-spheres using gauge theory. According to a recent result of Saveliev [100, these invariants fit together to give a candidate of another homomorphism on $\Theta_{\mathbb{Z}}^{3}$.

The situation being like this, $\Theta_{\mathbb{Z}}^{3}$ remains to be a rather mysterious group. Thus we have the following important problem. 
Problem 40. Study the central extension (20) from the point of view of group cohomology as well as geometric topology. In particular determine the Euler class of this central extension which is an element of the group

$$
H^{2}\left(\overline{\mathcal{H}}_{g, 1} ; \Theta_{\mathbb{Z}}^{3}\right) \cong \operatorname{Hom}\left(H_{2}\left(\overline{\mathcal{H}}_{g, 1}\right), \Theta_{\mathbb{Z}}^{3}\right) \oplus \operatorname{Ext}_{\mathbb{Z}}\left(H_{1}\left(\overline{\mathcal{H}}_{g, 1}\right), \Theta_{\mathbb{Z}}^{3}\right) .
$$

This should be an extremely difficult problem. Here we would like to indicate a possible method of attacking it, and in particular a possible way of obtaining additive invariants for the group $\Theta_{\mathbb{Z}}^{3}$, very briefly. Details will be given in a forthcoming paper.

Using the traces, we can define a series of certain cohomology classes

$$
\tilde{t}_{2 k+1} \in H^{2}\left(\lim _{\longleftarrow} \operatorname{Aut}_{0}\left(\Gamma / \Gamma_{m}\right)\right) \quad(k=1,2, \cdots) .
$$

These are the "group version" of the elements $t_{2 k+1} \in H^{2}\left(\mathfrak{h}_{g, 1}^{\mathbb{Q}}\right)^{S p}$ defined in $\S 6$ (Definition 26). The homomorphism $\sigma$ is trivial on $\Theta_{\mathbb{Z}}^{3}$ so that we have the induced homomorphism $\bar{\sigma}: \overline{\mathcal{H}}_{g, 1} \rightarrow \underset{\lim }{\longleftarrow} \operatorname{Aut}_{0}\left(\Gamma / \Gamma_{m}\right)$.

Conjecture 41.

1. $\bar{\sigma}^{*}\left(\tilde{t}_{2 k+1}\right)$ is non-trivial in $H^{2}\left(\overline{\mathcal{H}}_{g, 1}\right)$ for any $k$

2. $\sigma^{*}\left(\tilde{t}_{2 k+1}\right)$ is trivial in $H^{2}\left(\mathcal{H}_{g, 1}\right)$ for any $k$.

The first part of the above conjecture is the "group version" of Conjecture 27] and it should be even more difficult to prove. On the other hand, if the classes $\sigma^{*}\left(\tilde{t}_{2 k+1}\right)$ were non-trivial, then they would serve as invariants for certain 4-manifolds (2-dimensional family of homology cylinders). This seems unlikely to be the case. Thus the second part is related to the following problem.

Problem 42. Determine the abelianization of the group $\mathcal{H}_{g, 1}$. Is it trivial? Also determine the second homology group $H_{2}\left(\mathcal{H}_{g, 1} ; \mathbb{Z}\right)$. Is the rank of it equal to 1 given by the signature?

If everything will be as expected, we would obtain non-trivial homomorphisms

$$
\hat{t}_{2 k+1}: \Theta_{\mathbb{Z}}^{3} \longrightarrow \mathbb{Z}
$$

as secondary invariants associated to the cohomology classes $\tilde{t}_{2 k+1}$. There should be both similarity and difference between these cases and the situation where we interpreted the Casson invariant as the secondary invariant associated to the first Mumford-Morita-Miller class $e_{1}$ (see 74]). More precisely, they are similar because they are all related to some cohomology classes in $H^{2}\left(\mathfrak{h}_{g, 1}^{\mathbb{Q}}\right)$. They are different because $e_{1}$ is non-trivial in $H^{2}\left(\mathcal{H}_{g, 1}\right)$ whereas we expect that the other classes $\sigma^{*}\left(\tilde{t}_{2 k+1}\right)$ would be all trivial in the same group. 
Also recall here that Matumoto [67] and Galewsky and Stern [23] proved that every topological manifold (of dimension $n \geq 7$ ) is simplicially triangulable if and only if the homomorphism (21) splits. In view of this result, it should be important to investigate the mod 2 structure of the extension (20) keeping in mind the works of Birman and Craggs 3] as well as Johnson [44 for the case of the mapping class group.

However we have come too far and surely many things have to be clarified before we would understand the structure of the group $\mathcal{H}_{g, 1}$.

Finally we would like to propose a problem.

Problem 43. Generalize the infinitesimal presentation of the Torelli Lie algebra given by Hain 29] to the case of the group of homology cobordism classes of homology cylinders.

\section{DiffeOMORPhism GROUPS OF SURFACES}

Let us recall the important problem of constructing (and then detecting) characteristic classes of smooth fiber bundles as well as those of foliated fiber bundles whose fibers are diffeomorphic to a general closed $C^{\infty}$ manifold $M$. This is equivalent to the problem of computing the cohomology groups $H^{*}(\mathrm{BDiff} M)\left(\operatorname{resp} . H^{*}\left(\mathrm{BDiff}^{\delta} M\right)\right)$ of the classifying space of the diffeomorphim group Diff $M$ (resp. the same group $\operatorname{Diff}^{\delta} M$ equipped with the discrete topology) of $M$. Although the theory of higher torsion invariants of fiber bundles developed by Igusa 38 on the one hand and by Bismut, Lott 4 and Goette on the other and also the Gel'fand-Fuks cohomology $H_{G F}^{*}(M)$ of $M$ (see e.g. [28]) produce characteristic classes for the above two types of $M$-bundles, there seem to be only a few known results concerning explicit computations for specific manifolds. The same problems are also important for various subgroups of Diff $M$, in particular the symplectomorphism $\operatorname{group} \operatorname{Symp}(M, \omega)$ (in the case where there is given a symplectic form $\omega$ on $M)$ as well as the volume preserving diffeomorphism group.

Here we would like to propose two problems for the special, but at the same time very important case of surfaces. Note that we have two characteristic classes

$$
\int_{\text {fiber }} u_{1} c_{1}^{2}, \int_{\text {fiber }} u_{1} c_{2} \in H^{3}\left(\operatorname{BDiff}_{+}^{\delta} \Sigma_{g} ; \mathbb{R}\right)
$$

which are defined to be the fiber integral of the characteristic classes $u_{1} c_{1}^{2}, u_{1} c_{2}$ of codimention two foliations. In the case of $g=0$ (namely $S^{2}$ ), Thurston and then Rasmussen 94] (see also Boullay 8]) proved 
that these classes are linearly independent and vary continuously. Although it is highly likely that their results can be extended to the cases of surfaces of higher genera, explicit construction seems to be open.

Problem 44. Prove that the above characteristic classes induce surjective homomorphism

$$
H_{3}\left(\mathrm{BDiff}_{+}^{\delta} \Sigma_{g} ; \mathbb{Z}\right) \longrightarrow \mathbb{R}^{2}
$$

for any $g$.

The cohomology classes in (22) are stable with respect to $g$. On the other hand, in [57] [58] we found an interesting interaction between the twisted cohomology group of the mapping class group and some well known concepts in symplectic topology such as the flux homomorphism as well as the Calabi homomorphism (see 68 for generalities of the symplectic topology). By making use of this, we defined certain cohomology classes of BSymp ${ }^{\delta} \Sigma_{g}$ and proved non-triviality of them.

In view of the fact that all the known cohomology classes of BDiff ${ }_{+}^{\delta} \Sigma_{g}$ as well as $\operatorname{BSymp}^{\delta} \Sigma_{g}$ are stable with respect to the genus, we would like to ask the following problem.

Problem 45. Study whether the homology groups of BDiff ${ }_{+}^{\delta} \Sigma_{g}$ stabilize with respect to $g$ or not. The same problem for the group $\operatorname{Symp}^{\delta} \Sigma_{g}$.

Acknowledgments The author would like to express his hearty thanks to R. Hain, N. Kawazumi, D. Kotschick, M. Matsumoto, H. Nakamura, T. Sakasai for enlightening discussions as well as useful informations concerning the problems treated in this paper.

\section{REFERENCES}

1. S. Andreadakis, On the automorphisms of free groups and free nilpotent groups, Proc. London Math. Soc. 15 (1965), 239-268.

2. M. Bestvina, The topology of $\operatorname{Out}\left(F_{n}\right)$, Proceedings of the International Congress of Mathematicians, Beijing 2002, Higher Ed. Press, Beijing 2002, 373384 .

3. J. Birman and R. Craggs, The $\mu$-invariant of 3-manifolds and certain structural properties of the group of homeomorphisms of a closed oriented 2manifold, Trans. Amer. Math. Soc. 237 (1978), 283-309.

4. J.-M. Bismut and J. Lott, Flat vector bundles, direct images and higher real analytic torsion, J. Amer. Math. Soc. 8 (1995), 291-363.

5. D. Biss and B. Farb, $\mathcal{K}_{g}$ is not finitely generated, preprint. 
6. A. Borel, Stable real cohomology of arithmetic groups, Ann. Sci. École Norm. Sup. 7 (1974), 235-272.

7. A. Borel, Stable real cohomology of arithmetic groups II, in Manifolds and Groups, Papers in Honor of Yozo Matsushima, Progress in Math. 14, Birkhäuser, Boston, 1981, 21-55.

8. P. Boullay, $H_{3}\left(\right.$ Diff $\left.f^{+}\left(S^{2}\right) ; \mathbb{Z}\right)$ contains an uncountable $\mathbb{Q}$-vector space, Topology 35 (1996), 509-520.

9. J. Conant and K. Vogtmann, On a theorem of Kontsevich, Alg. Geom. Topology 3 (2003), 1167-1224.

10. J. Conant and K. Vogtmann, Morita classes in the homology of automorphism group of free groups, Geometry and Topology 8 (2004), 1471-1499.

11. M. Culler and K. Vogtmann, Moduli of graphs and automorphisms of free groups, Invent. Math. 84 (1986), 91-119.

12. C.J. Earle and J. Eells, The diffeomorphism group of a compact Riemann surface, Bull. Amer. Math. Soc. 73 (1967), 557-559.

13. P. Elbaz-Vincent, H. Gangl and C. Soulé, Quelques calculs de la cohomologie de $G L_{N}(\mathbb{Z})$ et la K-théorie de $\mathbb{Z}$, C. R. Math. Acad. Sci. Paris 335 (2002), $321-324$.

14. H. Endo, Meyer's signature cocycle and hyperelliptic fibrations, Math. Ann. 316 (2000), 237-257.

15. C. Faber, A conjectural description of the tautological ring of the moduli space of curves, in Moduli of Curves and Abelian Varieties, Carel Faber and Eduard Looijenga, editors, Vieweg 1999.

16. R. Fintushel and R. Stern, Instanton homology of Seifert fibred homology three spheres, Proc. London Math. Soc. 61 (1990), 109-137.

17. K. Fr $\phi y$ shov, Equivariant aspects of Yang-Mills Floer theory, Topology 41 (2002), 525-552.

18. Y. Fukumoto and M. Furuta, Homology 3-spheres bounding acyclic 4manifolds, Math. Res. Letters 7 (2000), 757-766.

19. Y. Fukumoto, M. Furuta and M. Ue, W-invariants and Neumann-Siebenmann invariants for Seifert homology 3-spheres, Topology Appl. 116 (2001), 333-369.

20. W. Fulton and J. Harris, Representation Theory, Graduate Texts in Math. 129, Springer Verlag 1991.

21. M. Furuta, Homology cobordism group of homology 3-spheres, Invent. Math. 100 (1990), 339-355.

22. S. Galatius, Mod $p$ homology of the stable mapping class group, Topology 43 (2004), 1105-1132.

23. D. Galewski and R. Stern, Classification of simplicial triangulations of topological maifolds, Ann. of Math. 111 (1980), 1-34.

24. S. Garoufalidis and J. Levine, Tree-level invariants of three-manifolds, Massey products and the Johnson homomorphism, in Graphs and Patterns in Mathematics and Theoretical Physics, Proc. Sympos. Pure Math. 73 (2005), 173-205.

25. F. Gerlits, Invariants in chain complexes of graphs, preprint.

26. M. Goussarov, Finite type invariants and n-equivalence of 3-manifolds, C. R. Math. Acad. Sci. Paris 329 (1999), 517-522.

27. K. Habiro, Claspers and finite type invariants of links, Geometry and Topology 4 (2000), 34-43. 
28. A. Haefliger, Sur les classes caractéristiques des feuilletages, Séminaire Bourbaki, 1971/72, Lecture Notes in Mathematics, vol. 317, Springer Verlag, 1973, 239-260.

29. R. Hain, Infinitesimal presentations of the Torelli groups, J. Amer. Math. Soc. 10 (1997), 597-651.

30. R. Hain and E. Looijenga, Mapping class groups and moduli space of curves, Proc. Symp. Pure Math. 62.2 (1997), 97-142.

31. R. Hain and M. Matsumoto, Weighted completion of the Galois groups and Galois actions on the fundamental group of $\mathbf{P}^{1}-\{0,1, \infty\}$, Compositio Math. 139 (2003), 119-167.

32. R. Hain and M. Matsumoto, Galois actions on fundamental groups of curves and the cycle $C-C_{-}$, to appear in J. Inst. Math. Jussieu.

33. R. Hain and D. Reed, On the Arakelov geometry of the moduli spaces of curves, Journal of Differential Geometry 67 (2004), 195-228.

34. J. Harer, Stability of the homology of the mapping class group of an orientable surface, Ann. of Math. 121 (1985), 215-249.

35. A. Hatcher, Homological stability for automorphism groups of free groups, Comment. Math. Helv. 70 (1995), 129-137.

36. A. Hatcher and K. Vogtmann, Rational homology of Aut $\left(F_{n}\right)$, Math. Res. Letters 5 (1998), 759-780.

37. A. Hatcher and K. Vogtmann, Homology stability for outer automorphism groups of free groups, Alg. Geom. Topology 4 (2004), 1253-1272.

38. K. Igusa, Higher Franz-Reidemeister Torsion, AMS/IP Studies in Advanced Mathematics, American Mathematical Society, 2002.

39. E-N. Ionel, Relations in the tautological ring of $\mathcal{M}_{g}$, preprint.

40. D. Johnson, An abelian quotient of the mapping class group $\mathcal{I}_{q}$, Math. Ann. 249 (1980), 225-242.

41. D. Johnson, A survey of the Torelli group, Contemporary Math. 20 (1983), 165-179.

42. D. Johnson, The structure of the Torelli group I: A finite set of generators for $\mathcal{I}_{g}$, Ann. of Math. 118 (1983), 423-442.

43. D. Johnson, The structure of the Torelli group II: A characterization of the group generated by twists on bounding simple closed curves, Topology 24 (1985), 113-126.

44. D. Johnson, The structure of the Torelli group III: The abelianization of $\mathcal{I}_{g}$, Topology 24 (1985), 127-144.

45. V. Jones, Hecke algebra representations of braid groups and link polynomials, Ann. of Math. 126 (1987), 335-388.

46. Y. Kasahara, An expansion of the Jones representation of genus 2 and the Torelli group, Alg. Geom. Topology 1, (2001), 39-55.

47. M. Kassabov, On the automorphism tower of free nilpotent groups, thesis, Yale University, 2003.

48. N. Kawazumi, A generalization of the Morita-Mumford classes to extended mapping class groups for surfaces, Invent. Math. 131 (1998), 137-149.

49. N. Kawazumi, Cohomological aspects of the Magnus expansions, preprint.

50. N. Kawazumi, Harmonic Magnus expansion on the universal family of Riemann surfaces, notes from several lectures. 
51. N. Kawazumi and S. Morita, The primary approximation to the cohomology of the moduli space of curves and cocycles for the stable characteristic classes, Math. Res. Letters 3 (1996), 629-641.

52. N. Kawazumi and S. Morita, The primary approximation to the cohomology of the moduli space of curves and cocycles for the Mumford-Morita-Miller classes, preprint.

53. F. Kirwan, Cohomology of moduli spaces, Proceedings of the International Congress of Mathematicians, Beijing 2002, Higher Ed. Press, Beijing 2002, 363-382.

54. T. Kitano, On the first Morita-Mumford class of surface bundles over $S^{1}$ and the Rochlin invariant, J. Knot Theory Ramifications 9 (2000), 179-186.

55. M. Kontsevich, Formal (non-)commutative symplectic geometry, in The Gelfand Mathematical Seminars 1990-1992, Birkhäuser Verlag 1993, 173-188.

56. M. Kontsevich, Feynman diagrams and low-dimensional topology, in Proceedings of the First European Congress of Mathematicians, Vol II, Paris 1992, Progress in Math. 120, Birkhäuser Verlag 1994, 97-121.

57. D. Kotschick and S. Morita, Signature of foliated surface bundles and the group of symplectomorphisms of surfaces, Topology 44 (2005), 131-149.

58. D. Kotschick and S. Morita, Characteristic classes of foliated surface bundles with area-preserving total holonomy, preprint.

59. J. Levine, Link concordance and algebraic closure, Comment. Math. Helv. 64 (1989), 236-255.

60. J. Levine, Homology cylinders: an enlargement of the mapping class group, Alg. Geom. Topology 1 (2001), 243-270.

61. E. Looijenga, Cohomology of $\mathcal{M}_{3}$ and $\mathcal{M}_{3}^{1}$, in Mapping Class Groups and Moduli Spaces of Riemann Surfaces, C.-F. Bödigheimer and R. Hain, editors, Contemporary Math. 150 (1993), 205-228.

62. E. Looijenga, Stable cohomology of the mapping class group with symplectic coefficients and of the universal Abel-Jacobi map, J. Algebraic Geometry 5 (1996), 135-150.

63. I. Madsen and U. Tillmann, The stable mapping class group and $Q\left(\mathbb{C} P^{\infty}\right)$, Invent. Math. 145 (2001), 509-544.

64. I. Madsen and M. Weiss, The stable moduli space of Riemann surfaces: Mumford's conjecture, preprint 2002.

65. M. Matsumoto, Galois representations on profinite braid groups on curves, J. Reine. Angew. Math. 474 (1996), 169-219.

66. M. Matsumoto, Arithmetic fundamental groups and the moduli of curves, School on Algebraic Geometry (Trieste 1999), ICTP Lecture Notes 1, Abdus Salam Int. Cent. Theoret. Phys., Trieste 2000, 355-383.

67. T. Matumoto, Triangulation of manifolds, Algebraic and Geometric Topology, Stanford 1976, Proc. Sympos. Pure Math. 32 (1978), Part 2, 3-6.

68. D. McDuff and D.A. Salamon, Introduction to Symplectic Topology, second edition, Oxford University Press, 1998.

69. G. Mess, The Torelli groups for genus 2 and 3 surfaces, Topology 31 (1992), $775-790$.

70. E.Y. Miller, The homology of the mapping class group, Journal of Differential Geometry 24 (1986), 1-14. 
71. T. Morifuji, On Meyer's function of hyperelliptic mapping class group, J. Math. Soc. Japan 55 (2003), 117-129.

72. S. Morita, Characteristic classes of surface bundles, Bull. Amer. Math. Soc. 11 (1984), 386-388.

73. S. Morita, Characteristic classes of surface bundles, Invent. Math. 90 (1987), 551-577.

74. S. Morita, Casson's invariant for homology 3-spheres and characteristic classes of surface bundles, Topology 28 (1989), 305-323.

75. S. Morita, On the structure of the Torelli group and the Casson invariant, Topology 30 (1991), 603-621.

76. S. Morita, Abelian quotients of subgroups of the mapping class group of surfaces, Duke Math. J. 70 (1993), 699-726.

77. S. Morita, The extension of Johnson's homomorphism from the Torelli group to the mapping class group, Invent. Math. 111 (1993), 197-224.

78. S. Morita, A linear representation of the mapping class group of orientable surfaces and characteristic classes of surface bundles, in Proceedings of the Taniguchi Symposium on Topology and Teichmülller Spaces held in Finland, July 1995, World Scientific 1996, 159-186.

79. S. Morita, Casson invariant, signature defect of framed manifolds and the secondary characteristic classes of surface bundles, Journal of Differential Geometry 47 (1997), 560-599.

80. S. Morita, Structure of the mapping class groups of surfaces: a survey and a prospect, Geometry and Topology Monographs 2 (1999), Proceedings of the Kirbyfest, 349-406.

81. S. Morita, Structure of the mapping class group and symplectic representation theory, l'Enseignement Math. Monographs 38 (2001), 577-596.

82. S. Morita, Generators for the tautological algebra of the moduli space of curves, Topology 42 (2003), 787-819.

83. S. Morita, Structure of derivation algebras of surfaces and its applications, in preparation.

84. J. Moser, On the volume forms, Trans. Amer. Math. Soc. 120 (1965), 286-294.

85. D. Mumford, Towards an enumerative geometry of the moduli space of curves, in Arithmetic and Geometry, Progress in Math. 36 (1983), 271-328.

86. H. Nakamura, Coupling of universal monodromy representations of GaloisTeichmüller modular group, Math. Ann. 304 (1996), 99-119.

87. H. Nakamura, Galois rigidity of profinite fundamental groups, Sugaku Expositions 10 (1997), 195-215.

88. H. Nakamura, Limits of Galois representations in fundamental groups along maximal degeneration of marked curves, I, Amer. Jour. Math. 121 (1999), $315-358$.

89. H. Nakamura, Limits of Galois representations in fundamental groups along maximal degeneration of marked curves, II, Arithmetic fundamental groups and noncommutative algebra, Berkeley 1999, Proc. Sympos. Pure Math. 70 (2002), Amer. Math. Soc., 43-78.

90. W. Neumann, An invariant of plumbed homology spheres, Topology Symposium, Siegen 1979, Lect. Notes in Math. 788, Springer, 1980, 125-144.

91. R. Ohashi, in preparation. 
92. P. Ozsváth and Z. Szabó, Absolutely graded Floer homologies and intersection forms for four-manifolds with boundary, Advances in Math. 173 (2003), 179261.

93. A. Pettet, The Johnson homomorphism and the second cohomology of $\mathrm{IA}_{n}$, preprint.

94. O. H. Rasmussen, Continuous variation of foliations in codimension two, Topology 19 (1980), 335-349.

95. T. Sakasai, The Johnson homomorphism and the third rational cohomology group of the Torelli group, Topology and its Applications 148 (2005), 83-111.

96. T. Sakasai, Homology cylinders and the acyclic closure of a free group, preprint.

97. T. Satoh, Twisted first homology groups of the automorphism group of a free group, to appear in J. Pure Appl. Alg.

98. T. Satoh, New obstructions to the surjectivity of the Johnson homomorphism of the automorphism group of a free group, preprint.

99. N. Saveliev, Floer homology of Brieskorn homology spheres, Journal of Differential Geometry 53 (1999), 15-87.

100. N. Saveliev, Fukumoto-Furuta invariants of plumbed homology 3-spheres, Pacific J. Math. 205 (2002), 465-490.

101. L. Siebenmann, On vanishing of the Rohlin invariant and nonfinitely amphicheiral homology 3-spheres, Topology Symposium, Siegen 1979, Lect. Notes in Math. 788, Springer, 1980, 172-222.

102. J. Stallings, Homology and central series of groups, Journal of Algebra 2 (1965), 170-181.

103. M. Suzuki, The Magnus representation of the Torelli group $\mathcal{I}_{g, 1}$ is not faithful for $g \geq 2$, Proc. Amer. Math. Soc. 130 (2002), 909-914.

104. U. Tillmann, On the homotopy of the stable mapping class group, Invent. Math. 130 (1997), 157-175.

105. R. Vakil, The moduli space of curves and its tautological ring, Notices of the Amer. Math. Soc. 50 (2003), 647-658.

106. K. Vogtmann, Automorphisms of free groups and Outer Space, Geometricae Dedicata 94 (2002), 1-31.

107. N. Wahl, From mapping class groups to automorphism groups of free groups, to appear in J. London Math.Soc.

Department of Mathematical Sciences, University of Tokyo, Komaba, TOKYO 153-8914, JAPAN

E-mail address: morita@ms.u-tokyo.ac.jp 